\title{
Singularity free dilaton-driven cosmologies and pre-little-bangs
}

\author{
Massimo Giovannini* \\ DAMTP, 21 Silver Street, Cambridge, CB3 9EW, United Kingdom \\ and CERN, CH-1211, Geneva 23, Switzerland
}

(Received 7 July 1998; published 24 March 1999)

\begin{abstract}
There are no reasons why the singularity in the growth of the dilaton coupling should not be regularized, in a string cosmological context, by the presence of classical inhomogeneities. We discuss a class of inhomogeneous dilaton-driven models whose curvature invariants are all bounded and regular in time and space. We prove that the non-space-like geodesics of these models are all complete in the sense that none of them reaches infinity for a finite value of the affine parameter. We conclude that our examples represent truly singularity-free solutions of the low-energy beta functions. We discuss some symmetries of the obtained solutions and we clarify their physical interpretation. We also give examples of solutions with spherical symmetry. In our scenario each physical quantity is everywhere defined in time and space, the big-bang singularity is replaced by a maximal curvature phase where the dilaton kinetic energy reaches its maximum. The maximal curvature is always smaller than one (in string units). The coupling constant is also smaller than one and it grows between two regimes of constant dilaton, implying, together with the symmetries of the solutions, that higher genus and higher curvature corrections are negligible. We argue that our examples describe the occurrence of "little bangs"'(i.e., high curvature phases which never develop physical singularities). They also suggest the possibility of an unexplored "pre-little-bang"' phase. [S0556-2821(99)06104-4]

PACS number(s): 98.80.Hw, 11.25.Mj, 98.80.Cq
\end{abstract}

\section{INTRODUCTION AND MOTIVATIONS}

If a consistent approach to the unification of gravitational and gauge interactions exists at all, it will certainly have important consequences for cosmology and high-energy physics. From a phenomenological point of view, we would demand, in this framework, convincing answers to many puzzling questions which arose in cosmology through the years. If such a theory exists, it will have to show how the inflationary paradigm can be naturally implemented [1], how the (general relativistic) singularities may be regularized [2], and which is the role played, in the early Universe, by the gauge fields. In other words, the theory of the big bang. A great effort has been made, in this direction, in the context of superstring inspired cosmological models. Quite interesting results were obtained. For example, it turned out that (super)inflationary phases with growing curvature and dilaton coupling are a direct consequence of the classical solutions of the low-energy beta functions $[3,4]$. New symmetries [such as the scale factor duality and the generalized $O(d, d)$ invariance] appeared to relate the ordinary decelerated Friedmann-Robertson-Walker expanding phases to their superinflationary counterparts [5]. Appealing mechanisms for the amplification of the vacuum fluctuations were exploited in order to derive various phenomenological implications of those models [6,7]. Of course pre-big-bang models also suffer from various problems [8]. Among them, it is certainly relevant to mention the problem of the space-time singularity [9].

\footnotetext{
*Present address: Institute of Cosmology, Department of Physics and Astronomy, Tufts University, Medford, Massachusetts 02155. Electronic address: m.giovannini@damtp.cam.ac.uk, massimo.giovannini@cern.ch, giovan@cosmos2.phy.Tufts.edu
}

The study of the cosmological solutions of the tree-level low-energy superstring effective action showed the occurrence of different types of cosmological singularities which seemed indeed quite difficult to regularize. For example, in the context of pre-big-bang models [10] it was argued that regular solutions of the tree level beta functions were very hard to find (if not impossible). The conclusion of those studies was that the only possible way in order to have regular solutions is to invoke the role played by the corrections to the tree-level action. In particular it is currently assumed that the corrections arising from the string tension expansion could regularize the curvature singularities appearing at the tree level [9].

In this paper we are going to explore a different approach to the singularity problem in the context of string cosmology. Namely, we will suppose, from the very beginning, that the Universe might have been inhomogeneous around the singularity and we will try to understand if an inhomogeneous phase could regularize the solutions of the low-energy beta functions. In fact the main limitations of previous numerical studies of string cosmological singularities was that the Universe was assumed to be completely homogeneous during all its stages of evolution.

In Ref. [13] it was shown that it is perfectly possible to find analytical examples of regular inhomogeneous solutions with growing dilaton coupling. Moreover those solutions were shown to be regular in the string frame but also in the Einstein frame [13]. The present investigation should be regarded as a completion and an extension of the one reported in Ref. [13]. In the past the possibility of regularizing the inhomogeneities through an inhomogeneous phase was marginally discussed [11]. The attempt was to apply an $O(d, d)$ transformation to a (generically singular) $(1+1)$-dimensional cosmological model in order to obtain a higher-dimensional (regular) model. In that investigation, 
however, no examples of inhomogeneous models with growing dilaton coupling were given and the obtained regular solutions contained an antisymmetric tensor field and an oscillating dilaton coupling whose energy density was not vanishing at large distances. No study of the geodesic completeness and no study of the possible occurrence of closed trapped surfaces has been performed. We want also to mention that recently (singular) inhomogeneous string cosmological models were studied from different points of view $[3,12]$.

Concerning the regularity properties of the solutions given in Ref. [13] our point is now very simple. By itself the regularity of the curvature invariants is not sufficient in order to state that a certain solution is free of singularities. It is well known that it is quite possible to construct regular solutions whose geodesics are not complete. A typical example of this behavior is an eternally inflating de Sitter model (with scale factor $a(t)=\exp [H t]$ ) which is clearly not geodesically complete [14] since the space-time has an edge (see also Ref. [15] for a discussion of the singularity properties in the context of inflationary models).

The absence of geodesic completeness can indicate two possible situations. Either the geodesics incompleteness signals a removable singularity (as in the de Sitter case) and then a regular extension of the space-time is possible, or it shows some true singularity of the space-time. Of course, if the singularity in the geodesics turns out to be removable, the question arises of which extension to choose among all the ones theoretically possible. It is in fact possible that the theoretical extensions do not meet the wanted physical features of the solutions. In the case of de Sitter space, for example, a reasonable extension of the inflating space-time would include an initial contracting phase.

In this paper we want to show that it is not only possible to find regular (inhomogeneous) string cosmological models without invoking the role played by the string curvature corrections, but, at the same time we want to show that the geodesics constructed from the solutions are all complete in the sense that they can be extended arbitrarily to any value of the affine parameter. We find that, before playing with the idea of an inhomogeneous bang described on the basis of low-energy beta functions, it is better, from a logical point of view, to make sure that the solutions we found are defined for any value of the affine parameter. If this is not the case, any kind of cosmological solution, even the most appealing one, should be discarded for the simple reason that, in spite of the absence of any pole in the curvature invariants, a boundary in the geodesics defines an unphysical region of the classical solution.

After having studied the geodesic completeness of our solutions we will move to the analysis of the Hawking theorems. We will try to understand which is the hypothesis of the singularity theorems which turn out to be violated and we will spend some time in discussing the possible absence, for some of our examples, of closed trapped (spacelike) surfaces. One of our conclusions will be that a complete formulation of the Hawking theorems (in the string frame) seems to be needed.

Finally we will try to relax the symmetries of our solu- tions by analyzing the case of spherical symmetry. This last part of our investigation is particularly relevant in the context of recent results claiming that the gravitational collapse of a stiff fluid can be used in order to study the fine-tuning properties of the pre-big-bang scenario [3]. We will prove that singular solutions (with linearly growing dilaton) in the string frame lead to regular solutions in the Einstein frame which is the one employed in Ref. [3].

In the context of general relativity the idea that cosmological singularities could have been regularized via the introduction of strong inhomogeneities received, in the past, a lot of attention. In particular it was shown that it is perfectly possible to find regular (inhomogeneous) solutions with sources whose energy-momentum tensor obey the equation of state of a (perfect) radiation fluid [16]. This solution was proven to be geodesically complete [17], meeting then all the requirements for a singularity free model. Moreover recently these ideas were further explored with various extensions aiming at more realistic cosmological scenarios [18]. For earlier discussions of inhomogeneous cosmological models in general relativity one can find useful sources of informations in Refs. [19,21]. Other useful references (not directly related to our paper but more to the graceful exit problem in singular string cosmological models) can be found in Ref. [22].

Our paper is organized as follows. In Sec. II we will review the main notations and the basic equations involved in the study of inhomogeneous string cosmological models. In Sec. III we will study the geodesic completeness of our solutions whereas in Sec. IV we will contrast the behavior of our solutions with the classical singularity theorems. In Sec. $\mathrm{V}$ we will give some examples of regular spherically symmetric string cosmological backgrounds admitting a prelittle-bang phase and in Sec. VI we will come to our concluding remarks.

\section{INHOMOGENEOUS DYNAMICS OF PRE-LITTLE- BANGS}

There are no reasons why a phase of growing dilaton coupling should not be compatible with a highly inhomogeneous phase sufficiently close to the big-bang singularity. In string theory the maximal curvature scale is related to the inverse of the string length $\left(\lambda_{s}^{-1}\right)$ and the question is if we are allowed to describe such a regime with the tree-level effective action. The answer to this question is of course yes, provided the curvature invariants will stay small, in string units, all along the dynamical evolution. The second physical requirement one has to demand is that not only the curvature invariants are bounded but also that the string coupling will stay much smaller than 1 . If this would not be the case, we should add to the effective action also the loop (genus) corrections. In the context of the pre-big-bang scenario the bigbang was always associated with the maximal curvature scale of the model. In that context the dynamics of the bang in rooted in the presence of higher order curvature corrections.

In our context the dynamics of the bang (i.e., the mechanism able to regularize the curvature) is connected with the 
presence of inhomogeneities which allow a completely smooth evolution between two asymptotic regimes of constant dilaton coupling. Moreover, recently, the dynamics of the pre-big-bang models was analyzed using interesting numerical techniques and it has been argued that starting from quite general "asymptotically trivial" initial conditions certain portions of the parameter space will collapse towards a singularity where the string tension corrections will hopefully [9] trigger a constant curvature phase. We remind the reader that these studies also foresee that other regions of the initial parameter space will not collapse towards a singularity. In spite of the fact that in Ref. [3] the spherical symmetry was always assumed in order to discuss the past asymptotically trivial state according to our picture it is perfectly possible that starting from a regime of small curvature and couplings some regions will not end up in a singularity but in a perfectly regular background whose maximal curvature can be as large as 0.1 (in string units). We will name these physical situations "little bang." The bang occurs because the curvature scale reaches a maximal value of the order of (but smaller than) $\lambda_{s}^{-2}$. At the same time the bang is small if compared to the typical (singular) bangs taking place in the scenarios of [3]. In Sec. V we will elaborate more on the role played by spherical symmetry in the context of little bangs.

Consider the low-energy string effective action in the absence of any dilaton potential

$$
S=-\frac{1}{\lambda_{s}^{2}} \int d^{4} x \sqrt{-g} e^{-\phi}\left[R+g^{\alpha \beta} \partial_{\alpha} \phi \partial_{\beta} \phi\right] .
$$

We deal, more specifically, with the case where the antisymmetric tensor field has been set to zero. In this approximation the tree-level beta functions can be obtained by direct variation of the action with respect to the metric tensor and to the dilaton field. After a linear combination of the obtained equations we have

$$
\begin{gathered}
R-g^{\alpha \beta} \partial_{\alpha} \phi \partial_{\beta} \phi+2 \square \phi=0, \\
R_{\mu}^{\nu}+\nabla_{\mu} \nabla^{\nu} \phi=0 .
\end{gathered}
$$

We consider now the line element of an inhomogeneous cosmological model with two spacelike Killing vector fields which admit an Abelian group $G_{2}$ on spacelike orbits $S_{2}$ [23]:

$$
d s^{2}=A(x, t)\left[d t^{2}-d x^{2}\right]-B(x, t)\left[C(x, t) d y^{2}+\frac{d z^{2}}{C(x, t)}\right] .
$$

Assuming that the space-time dependence of the metric functions can be factorized as

$$
\begin{aligned}
& A(x, t)=a(t) \alpha(x), \\
& B(x, t)=b(t) \beta(x), \\
& C(x, t)=c(t) \kappa(x),
\end{aligned}
$$

there can be a solution of the low-energy beta functions [13], provided

$$
\begin{aligned}
& A(x, t)=e^{\alpha g d(\mu t)}[\cosh \mu t]^{2+\beta}\left[\cosh \left(\frac{\mu x}{2}\right)\right]^{2 \beta(\beta+1)}, \\
& B(x, t)=e^{\alpha g d(\mu t)} \cosh \mu t \sinh \mu x \\
& C(x, t)=[\cosh \mu t]^{1+\beta}\left[\sinh \left(\frac{\mu x}{2}\right)\right]\left[\cosh \left(\frac{\mu x}{2}\right)\right]^{1+2 \beta},
\end{aligned}
$$

and

$$
\phi(t)=\alpha g d(\mu t)+\gamma,
$$

where

$$
g d(\mu t)=\arctan [\sinh \mu t]
$$

is the "Gudermannian" $[13,20]$ or hyperbolic amplitude and $\gamma$ is a numerical constant. This solution has two obvious symmetries which are also present in the original action, namely, it is invariant under dilaton shift $(\phi \rightarrow \phi$ + constant) and coordinate reparametrization $\left(x_{\mu} \rightarrow \mu x_{\mu}\right)$.

In terms of this symmetry the physical interpretation of the solution given in Eqs. (2.6) and (2.7) is very simple. The parameter $\mu$ has the dimensions of $[L]^{-1}$ and it fixes, in string units, the maximal curvature scale accessible. Since we demand regular solutions below the string scale, we have to require that $\mu<\lambda_{s}^{-1}$. In the case $\mu>\lambda_{s}^{-1}$ we always have regular solutions, but, in this regime curvature corrections will become leading. Since $\beta=\sqrt{\alpha^{2}+4}$ the only other physical parameter of the solution is exactly $\alpha$. Again the physical interpretation of this second parameter is quite simple: it measures, in string units, the maximal energy of the dilaton background since $\mu^{2} \alpha^{2}=\dot{\varphi}^{2}(0)$ (where the overdot stands for a derivation with respect to $t$ ).

There are "accidental" symmetries in the solution given in Eqs. (2.6), (2.7). We find that the metric is left invariant by a parity transformation and, therefore, given a solution and sending $x \rightarrow-x$ we obtain again a solution of the lowenergy beta functions. There is also another useful symmetry we want to mention. Namely, if $\beta \rightarrow-\beta$ and, at the same time, $\alpha \rightarrow-\alpha$ we get another solution of the low-energy beta functions. If the original solution had growing coupling, the transformed one will have decreasing coupling. Notice that this symmetry seems to be connected to the inhomogeneous dynamics of the solution more than with the original symmetry of the theory.

An interesting observation is that, since this family of solutions has regular curvature invariants for every value of $t$ the only curvature singularities may appear for $x=0$. The choice made in the derivation given in Eqs. (4.2) and (4.4) of Ref. [13] eliminates this possibility.

\section{GEODESIC COMPLETENESS}

In our previous discussion we did not claim that the solutions given in Eqs. (2.6) and (2.7) were singularity free and 
we always spoke about "regular" solutions. We could in principle have manifolds whose curvature invariants are regular but whose non-space-like geodesics reach a singularity in a finite proper time. This property of singular manifolds is generically called geodesic incompleteness and it means that the space-time contains non-space-like (i.e., either timelike or null) geodesics which (when maximally extended) have no end point in the regular part of the manifold.

If every non-space-like geodesic can be extended to arbitrary values of its affine parameter, then we can say that the manifold is geodesically complete. The purpose of the present section is to show that the solution given in Eqs. (2.6) and (2.7) is geodesically complete. To do this we have to show that every non-space-like geodesic has no end points. In other words we have to show that non-space-like geodesics can be extended to any arbitrary values of their affine parameter.

The first step in order to undertake this program is to write down the geodesics associated with the metric (2.6): namely,

$$
\begin{aligned}
& t^{\prime \prime}+\frac{\mu}{2}\left\{\frac{\alpha+(\beta+2) \sinh \mu t}{\cosh \mu t}\right\}\left[t^{\prime 2}+x^{\prime 2}\right] \\
& +\left\{\mu \beta(\beta+1) \tanh \left(\frac{\mu x}{2}\right)\right\} x^{\prime} t^{\prime} \\
& +\mu\left\{\frac{[\sinh (\mu x / 2)]^{2}[\alpha+(\beta+2) \sinh \mu t]}{[\cosh (\mu x / 2)]^{2 \beta^{2}-2} \cosh \mu t}\right\} y^{\prime 2} \\
& +\mu\left\{\frac{\alpha-\beta \sinh \mu t}{[\cosh (\mu x / 2)]^{4 \beta+2 \beta^{2}}[\cosh \mu t]^{2 \beta+3}}\right\} z^{\prime 2}=0, \\
& x^{\prime \prime}+\frac{\mu}{2}\left\{\beta(\beta+1) \tanh \left(\frac{\mu x}{2}\right)\right\}\left[t^{\prime 2}+x^{\prime 2}\right] \\
& +\mu\left\{\frac{\alpha+(\beta+2) \sinh \mu t}{\cosh \mu t}\right\} x^{\prime} t^{\prime} \\
& +\frac{\mu}{4}\left\{\sinh \mu x \frac{[\beta-(\beta+2) \cosh \mu x]}{[\cosh (\mu x / 2)]^{2 \beta^{2}}}\right\} y^{\prime 2} \\
& +\mu\left\{\beta \tanh \left(\frac{\mu x}{2}\right) \frac{[\cosh (\mu x / 2)]^{-4 \beta-2 \beta^{2}}}{[\cosh \mu]^{2(\beta+1)}}\right\} z^{\prime 2}=0 \text {, } \\
& y^{\prime \prime}+\mu\left\{\frac{[\alpha+(\beta+2) \sinh \mu t]}{\cosh \mu t}\right\} y^{\prime} t^{\prime} \\
& +\mu\left\{\frac{[(\beta+2) \cosh \mu x-\beta]}{\sinh \mu x}\right\} y^{\prime} x^{\prime}=0, \\
& z^{\prime \prime}+\mu\left\{\frac{[\alpha-\beta \sinh \mu t]}{\cosh \mu t}\right\} z^{\prime} t^{\prime}-\mu\left\{\beta \tanh \left(\frac{\mu x}{2}\right)\right\} x^{\prime} z^{\prime}=0,
\end{aligned}
$$

$$
\begin{aligned}
& {\left[t^{\prime 2}-x^{\prime 2}\right]-2\left\{\left[\cosh \left(\frac{\mu x}{2}\right)\right]^{2\left(1-\beta^{2}\right)}\right.} \\
& \left.\quad \times\left[\sinh \left(\frac{\mu x}{2}\right)\right]^{2}\right\} y^{\prime 2} \\
& \quad-2\left\{\left[\cosh \left(\frac{\mu x}{2}\right)\right]^{-2 \beta(\beta+2)}[\cosh \mu t]^{-2 \beta-2}\right\} z^{\prime 2} \\
& =\mathbf{s} e^{-\alpha g d(\mu t)}\left\{[\cosh \mu t]^{-(2+\beta)}\left[\cosh \frac{\mu x}{2}\right]^{-2 \beta(\beta+1)}\right\} .
\end{aligned}
$$

These equations can be easily derived once the Christoffel symbols have been computed (see Appendix A for more details). Notice that we shall be interested in the geodesics of the model in the string frame since, from the physical point of view, there are various reasons to prefer the string frame to the Einstein frame (see also Sec. IV about this point).

The previous system of coupled differential equations can be a bit simplified by integrating once Eqs. (3.3) and (3.4). The result is that Eqs. (3.2), (3.1), and (3.5) are left unchanged, whereas Eqs. (3.3) and (3.4) become first order:

$$
\begin{gathered}
y^{\prime} e^{\alpha g d(\mu t)}[\cosh \mu t]^{\beta+2}[\sinh \mu x]^{\beta+2}\left[\tanh \left(\frac{\mu x}{2}\right)\right]^{-\beta}=\mathbf{p}, \\
z^{\prime} e^{\alpha g d(\mu t)}[\cosh \mu t]^{-\beta}\left[\cosh \left(\frac{\mu x}{2}\right)\right]^{-2 \beta}=\mathbf{q}, \\
\mathbf{p}=\text { const, } \quad \mathbf{q}=\text { const. }
\end{gathered}
$$

Notice that $\mathbf{p}$ and $\mathbf{q}$ are integration constants whereas $\mathbf{s}$ is nothing but a parameter which takes values 0 for null geodesics and 1 for timelike geodesics. We will be mostly concerned with the cases of non-space-like geodesics. Before starting the analysis of the completeness of the geodesics we want to remind the reader of a standard result of the theory of ordinary differential equations [24]. Consider a generic first order differential equation of the form

$$
\frac{d f}{d v}=\mathcal{A}(f, v),
$$

and suppose that $\mathcal{A}(v)$ is a continuously differentiable functions of the variable $v$ defined in some interval $\mathcal{D}$. Then the solution of this equation exists in the whole interval where $v$ is defined provided it can be shown that $|d f(v) / d v|$ (i.e., the norm of the first derivative) is always finite for any finite value of $v$, which, in our case is nothing but the affine parameter. The fact that the geodesics are never singular for any finite value of the affine parameter, will imply that the geodesics are complete. Therefore the exercise we intend to do is to study the space of the solutions of the geodesics equations and to show that the solutions never show up singularities at finite $\lambda$. We will study future directed geodesics. Namely, we will suppose that the initial condition for the 
coupled system of differential equations are given at some finite value of $\lambda$ (which is arbitrary) and we will follow the geodesics forward in time. Of course one could choose to integrate the equations backward in time and the analysis turns out to be very similar. We will just report our calculations in the case of geodesics propagating towards the future. In order to show how to use the argument we just stated, we analyze, as a warm-up, a particularly simple case. Consider, for instance, the timelike geodesics of a point particle which is moving radially (i.e., $y^{\prime}=0, z^{\prime}=0, x^{\prime}=0$ ). In this case we have that Eq. (3.1) gives

$$
\frac{d g}{d t}+\frac{\mu}{2}\left[\frac{\alpha+(\beta+2) \sinh \mu t}{\cosh \mu t}\right] g=0, \quad g=\frac{d t}{d \lambda} .
$$

Trivial integration gives us that

$$
\frac{d t}{d \lambda}=e^{-(\alpha / 2) g d(\mu t)}[\cosh \mu t]^{-(\beta+2) / 2}
$$

[notice that Eq. (3.5) implies that the geodesics will be along $r=0]$. It is now clear that since $g d(\mu t)$ is a limited function and since $\beta=\sqrt{\alpha^{2}+4}>2$, then we have to conclude that

$$
\frac{d t}{d \lambda}<e^{-\pi \alpha / 4}=\text { const }
$$

The fact that $t^{\prime}$ is bounded by a constant implies that, in this specific case, that radial timelike geodesics are complete. In the two following subsections we will examine all the possible cases and we will then conclude that according to the argument we just discussed that the non-space-like geodesics of this metric are all complete.

\section{A. Lightlike geodesics: $s=0$}

Consider now the case of lightlike geodesics with $y^{\prime}$ $=z^{\prime}=0$. Direct integration of Eqs. (3.1) and (3.5) gives

$$
\begin{gathered}
x^{\prime}=\mathbf{u} e^{-\alpha g d(\mu t)}[\cosh \mu t]^{-(\beta+2)}\left[\cosh \left(\frac{\mu x}{2}\right)\right]^{-2 \beta(\beta+1)}, \\
t^{\prime}=\left|x^{\prime}\right|
\end{gathered}
$$

where $\mathbf{u}$ is nothing but an integration constant. Remember that in our solution $\beta= \pm \sqrt{\alpha^{2}+4}$. This implies that either we have $\beta<-2$ or $\beta>2$. If $\beta>2$ we have that $x^{\prime}$ $<\exp [-\pi \alpha / 2]$ which proves the completeness of the geodesics. If $\beta<-2$ we must conclude that since $x^{\prime}$ is always finite for any finite value of $\lambda$ the geodesics are also complete. A similar situation occurs in the case of lightlike geodesics with $x^{\prime}=y^{\prime}=0, x=0$ where direct integration of Eqs. (3.1) and (3.4) gives

$$
\begin{aligned}
& z^{\prime}=\mathbf{q} e^{-\alpha g d(\mu t)}[\cosh \mu t]^{\beta}, \\
& t^{\prime}=|\mathbf{q}| \sqrt{2} e^{-\alpha g d(\mu t)}[\cosh \mu t]^{-1} .
\end{aligned}
$$

In this case $t^{\prime}<\sqrt{2}|\mathbf{q}| \exp [-\pi \alpha / 2]$ for any value of $\beta$ and $z^{\prime}$ is also finite for any finite value of $\lambda$.

We want now to explore the case of lightlike geodesics in the case $y^{\prime}=0$. In order to do this we go to Eqs. (3.1) and (3.5). Moreover, from Eq. (3.5) we can see that it is possible to introduce a new variable which we call $w$. In terms of this new variable, Eqs. (3.5) and (3.7) reduce to

$$
\begin{aligned}
& \frac{d t}{d \lambda}=\cosh w \mathcal{F}(x, t), \\
& \frac{d x}{d \lambda}=\sinh w \mathcal{F}(x, t), \\
& \frac{d w}{d \lambda}=\mathcal{G}(w, x, t) \mathcal{F}(x, t),
\end{aligned}
$$

where

$$
\begin{aligned}
\mathcal{F}(x, t)=|\mathbf{q}| & \sqrt{2} e^{-\alpha g d \mu t}\left[\cosh \left(\frac{\mu x}{2}\right)\right]^{-\beta^{2}}[\cosh \mu t]^{-1}, \\
\mathcal{G}(w, x, t)= & -\left[\frac{\mu}{2} \beta(\beta+2) \tanh \left(\frac{\mu x}{2}\right) \cosh w\right. \\
& +\mu(\beta+1) \tanh \mu t \sinh w] .
\end{aligned}
$$

The equation for $w^{\prime}$ can be obtained in the following way. From Eq. (3.5) we can express $z^{\prime 2}$ in terms of $t^{\prime 2}$ and $x^{\prime 2}$. Then we can go either to Eq. (3.1) or to Eq. (3.2). Let us focus, for instance, on Eq. (3.1). We can notice that by taking the derivative of $t^{\prime}$ with respect to the affine parameter and by using Eqs. (3.15), (3.16) $t^{\prime \prime}$ can be expressed as

$$
\begin{aligned}
t^{\prime \prime}= & w^{\prime} \sinh w \mathcal{F}(x, t)-\left[\mu\left(\frac{\alpha+\sinh \mu t}{\cosh \mu t}\right) \cosh ^{2} w\right. \\
& \left.+\frac{\mu}{2} \beta^{2} \tanh \left(\frac{\mu x}{2}\right) \cosh w \sinh w\right] \mathcal{F}^{2}(x, t) .
\end{aligned}
$$

By inserting the $t^{\prime \prime}$ obtained from this last equation into Eq. (3.1) we get exactly the evolution for $w^{\prime}$ reported in Eqs. (3.15), (3.16). We will use again the same procedure in the following formulas but we will not repeat this derivation. The evolution for $z^{\prime}$ is simply given by Eq. (3.7) and does not need to be rewritten. Notice, first of all, that the sign of $\mathcal{F}(x, t)$ is always positive for every value of $\mathbf{q}$ [which is the integration constant appearing in Eq. (3.7)]. Suppose that $x^{\prime}>0$. In this case we have that, since $x(\lambda)$ and $t(\lambda)$ are two increasing functions of the affine parameter, the sign of $w^{\prime}$ is only determined by the relative weight of the numerical constants appearing in front of the two hyperbolic tangents contained in the expression of $\mathcal{G}(w, x, t)$. Now, since the curvature invariants are bounded provided $|\beta|>2$ we have that the sign of $\mathcal{G}(w, x, t)$ is always negative, and that, therefore, $w(\lambda)$ increases without being singular for any finite $\lambda$. Thus $w(\lambda)$ does not diverge for finite $\lambda$ and since $x^{\prime}$ cannot grow arbitrarily large we have to conclude that the geodesics are 
all complete. Suppose now that $x^{\prime}<0$. This time we have that, since $\tanh (\mu x / 2)$ always decreases as a function of $\lambda$, the sign of $w^{\prime}$ will be determined by the sign of $-\mu(\beta$ +1 ) which is positive (for large $\lambda$ ) if $\beta<-2$ and negative for $\beta>2$. In both cases $w^{\prime}$ is always finite. It is also easy to see that this system of equations does not yield singularities when the geodesics approach $x\left(x^{\prime}<0, \sinh w<0\right)$. The reason is that $w^{\prime}$ becomes positive and prevents the $x$ coordinate from decreasing too quickly. The same argument can be used in the case of the $t$ coordinate. If $t$ would grow too fast this would imply that $x$ would grow or decrease too quickly. But then the action of $w^{\prime}$ will again prevent possible divergences. In Figs. 1 and 2 we report some plots of the geodesics studied in this last case [Eqs. (3.15)]. We report the plots only for illustration and in order to corroborate with some picture the analytical arguments presented up to now.

We can easily integrate the geodesics also in the case where $z^{\prime}=0$. We will have

$$
\begin{aligned}
& \frac{d t}{d \lambda}=\cosh w \mathcal{M}(x, t), \\
& \frac{d x}{d \lambda}=\sinh w \mathcal{M}(x, t), \\
& \frac{d w}{d \lambda}=\mathcal{M}(x, t) \mathcal{N}(w, x, t),
\end{aligned}
$$

where

$$
\begin{aligned}
\mathcal{M}(x, t)= & \mathbf{p} \sqrt{2} e^{-\alpha g d(\mu t)} \\
& \times \frac{[\cosh (\mu x / 2)]^{1-\beta-\beta^{2}}[\sinh (\mu x / 2)]^{1+\beta}}{[\sinh \mu x]^{(\beta+2)}[\cosh \mu t]^{(\beta+2)}}, \\
\mathcal{N}(w, x, t)= & -\frac{\mu}{2} \cosh w \\
& \times\left\{\left(\beta^{2}-1\right) \tanh \left(\frac{\mu x}{2}\right)-\left[\tanh \left(\frac{\mu x}{2}\right)\right]^{-1}\right\} .
\end{aligned}
$$

In this case the situation seems a little bit more complicated. Consider, in particular the analytic expression of $\mathcal{M}(x, t)$. We can immediately see that for $x \rightarrow 0, \mathcal{M}(x, t)$ might diverge as soon as $x=0$ is reached. Therefore we have first of all to understand if $x=0$ can be ever approached by the geodesics. In order to do this we can divide $x^{\prime}$ by $w^{\prime}$ and find an analytical relation between $w$ and $x$. The differential relation is

$$
\frac{d x}{d w}=-\frac{2}{\mu} \frac{\tanh w}{\left(\beta^{2}-1\right) \tanh (\mu x / 2)-[\tanh (\mu x / 2)]^{-1}} .
$$

By integrating this equation once we find that
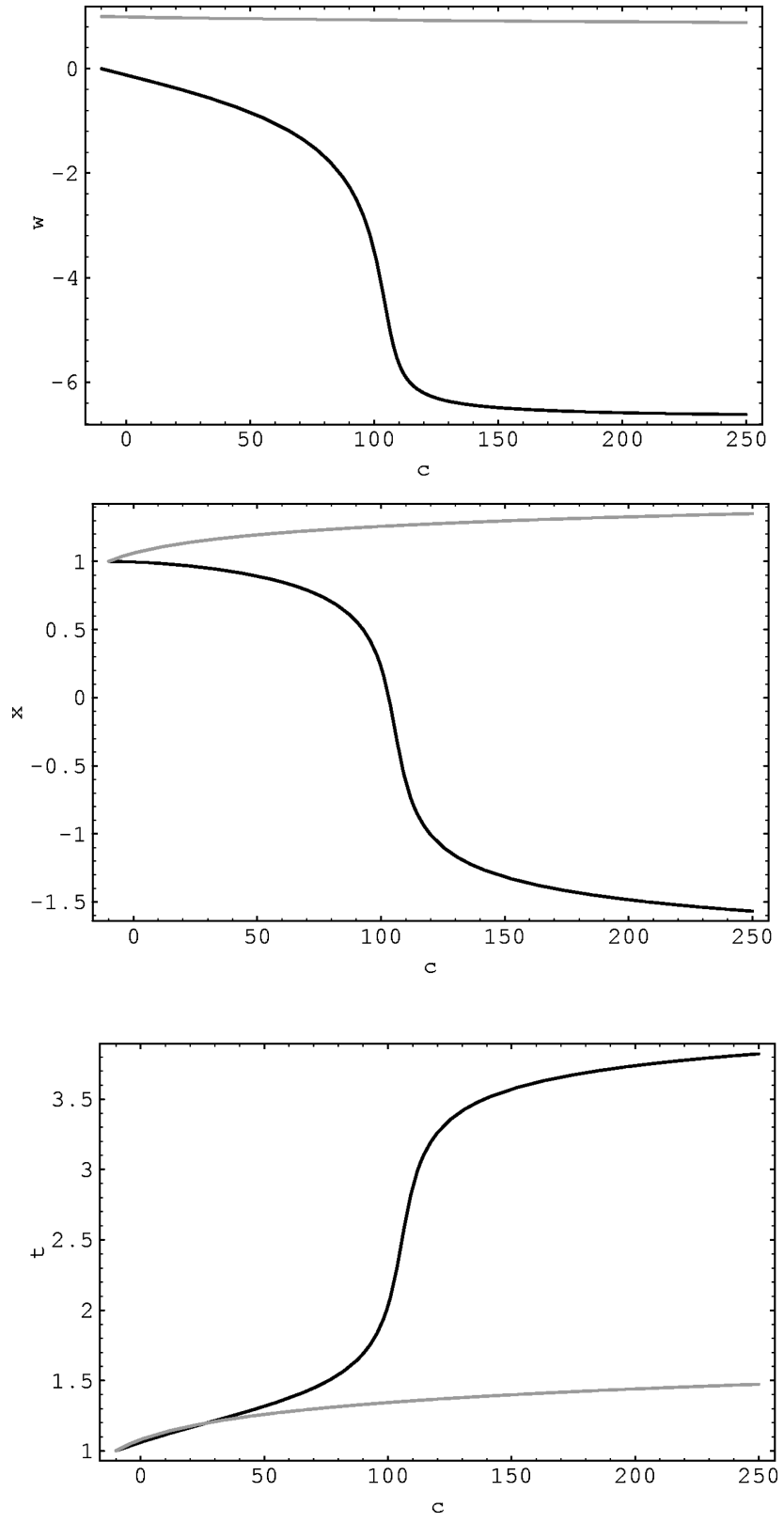

FIG. 1. We report some examples of light-like geodesics which are solutions of the system of differential equations given in Eq. (3.15). In this case $\beta=-4$ and we took the initial conditions to be $x(-10)=1, t(-10)=1$, and $w(-10)=0$ (full curves) and $x$ $(-10)=1, t(-10)=1, w(-10)=1$ (light curves). On the horizontal axis is reported the affine parameter $\lambda=c$, whereas on the vertical axes are reported, in each figure, the various geodesics and the auxiliary function $w$. We can clearly see that $t^{\prime}$ is always positive. If $x^{\prime}<0$ then we expect that $w^{\prime}<0$ (since $\beta<-2$ ). On the other hand, if $x^{\prime}>0$ we expect that $w^{\prime}<0$. In both cases no singularities are present for finite $c$.

$$
\cosh w=\mathbf{k}^{-1} \sinh \left(\frac{\mu x}{2}\right)\left[\cosh \left(\frac{\mu x}{2}\right)\right]^{1-\beta^{2}} .
$$

Notice that $\mathbf{k}$ is a positive integration constant. From this expression we learn that the solution of the system given in Eqs. (3.17) will never pass through $x=0$. In fact we can 

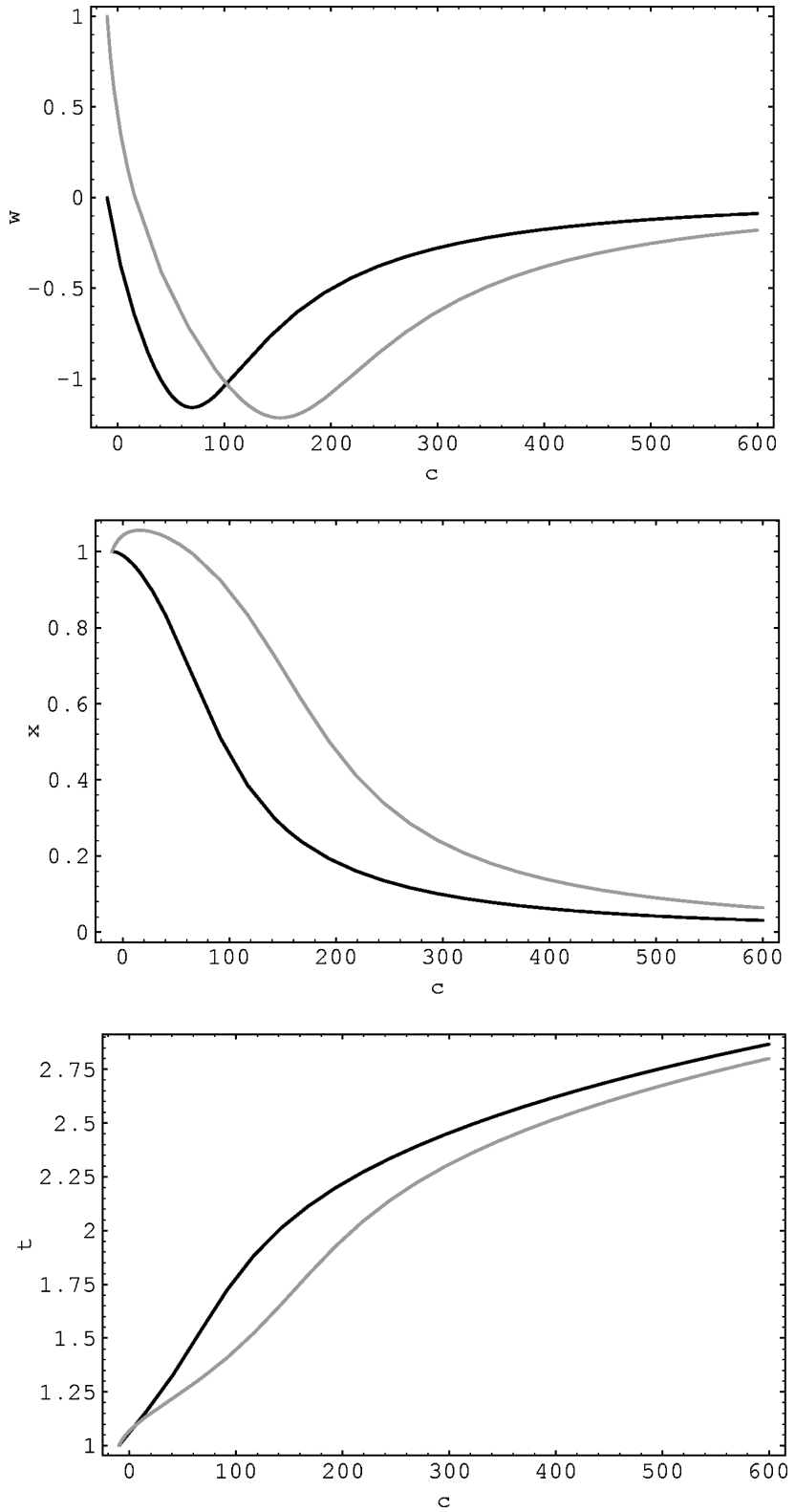

FIG. 2. We report the solutions of the system given in Eqs. (3.15) with the same initial conditions discussed in the previous figure for light and full curves, but with $\beta=4$.

notice that $\mathbf{k} \cosh w \geqslant \mathbf{k}$. But if $x=0$, then, the right-hand side of Eq. (3.20) will also go to zero and this will mean that $\mathbf{k} \cosh w$ has to go to zero which is never the case. We can visualize this fact by plotting the function

$$
f(x)=\sinh \left(\frac{\mu x}{2}\right)\left[\cosh \left(\frac{\mu x}{2}\right)\right]^{1-\beta^{2}}
$$

In Fig. 3 we report this plot. We notice that for each particular value of $\mathbf{k}$ there will be two intersections with the curve $f(x)$ (corresponding to the case $w=0$ ). This implies that $x$ is always positive and its variation will be bounded between these two intersections. This implies that $\mathcal{M}(x, t)$ will also be positive definite. Since according to the above relation

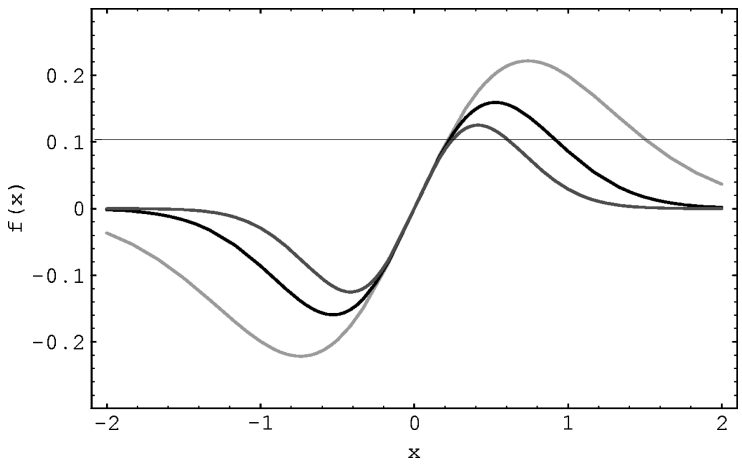

FIG. 3. We plot the function $f(x)$ given in Eq. (3.21). The three curves from top to bottom correspond, respectively, to the cases $\beta=3$, 4, 5. Notice that in Eq. (3.20) the dependence on $\beta$ only occurs through the factor $\left(1-\beta^{2}\right)$ and it is, therefore, invariant for $\beta \rightarrow-\beta$. With the horizontal line is denoted a particular value of $\mathbf{k}$.

(which has to hold for any $\lambda$ ) $\cosh w$ will never be zero (by its definition), $x(\lambda)$ will never reach zero, and $t(\lambda)$ will be an increasing function of the affine parameter which never gets singular for any finite value of $\lambda$. Moreover the $x$ coordinate can only take values between $x_{+}$and $x_{-}$where $x_{ \pm}$are defined from the solution of the transcendent equation $f\left(x_{ \pm}\right)=\mathbf{k}$. The same argument shows that $x^{\prime}$ is also finite for any finite value of $\lambda$ and this implies that the geodesics are complete.

\section{B. Timelike geodesics: $s=1$}

Along the lines of this last case we can treat the case where $y^{\prime}=0, z^{\prime}=0$ and $\mathbf{s}=1$. In this case it is again useful to introduce the variable $w$. From Eqs. (3.5) and (3.6) we can see that

$$
\begin{aligned}
& \frac{d t}{d \lambda}=\cosh w \quad \mathcal{P}(x, t), \\
& \frac{d x}{d \lambda}=\sinh w \mathcal{P}(x, t), \\
& \frac{d w}{d \lambda}=\mathcal{R}(w, x, t) \mathcal{P}(x, t),
\end{aligned}
$$

where

$$
\begin{aligned}
\mathcal{P}(x, t)= & \sqrt{\mathbf{s}} e^{-(\alpha / 2) g d(\mu t)} \\
& \times[\cosh \mu t]^{-(\beta+2) / 2}\left[\cosh \left(\frac{\mu x}{2}\right)\right]^{-\beta(\beta+1)}, \\
\mathcal{R}(w, x, t)= & -\frac{\mu}{2}\left[\frac{\alpha+(\beta+2) \sinh \mu t}{\cosh \mu t} \sinh w\right. \\
& \left.+\beta(\beta+1) \tanh \left(\frac{\mu x}{2}\right) \cosh w\right] .
\end{aligned}
$$

From Eqs. (3.22) and (3.23) we can see that $t^{\prime}$ is always positive for time-like geodesics and it is also finite for any $\beta$. 
Suppose now that $x^{\prime}>0$. Then the sign of $w^{\prime}$ will be mainly determined by the sign of $\mathcal{R}(w, x, t)$. Since $x(\lambda)$ and $t(\lambda)$ are always increasing functions of the affine parameter we have that $w(\lambda)$ increases, without being singular, for any finite $\lambda$. Then the geodesics are all complete. With a similar argument we can prove the completeness in the case $x^{\prime}<0$.

If $\mathbf{s}=1$ we have also to study the case $x^{\prime}=0, y^{\prime}=0, x$ $=0$. In this case from Eq. (3.4) we have that the evolution equations for Eqs. (3.4) and (3.1) can be written as

$$
\begin{aligned}
\frac{d z}{d \lambda}= & \mathbf{q} e^{-\alpha g d(\mu t)}[\cosh \mu t]^{\beta}, \\
\frac{d t}{d \lambda}= & {[\cosh \mu t]^{-1}\left\{2 \mathbf{q}^{2} e^{-2 \alpha g d(\mu t)}\right.} \\
& \left.+\mathbf{s} e^{-\alpha g d(\mu t)}[\cosh \mu t]^{-\beta}\right\}^{1 / 2} .
\end{aligned}
$$

Again in this case we see that $z^{\prime}$ and $t^{\prime}$ are always finite and no singularity is encountered for any finite $\lambda$. Moreover, suppose that $\beta>2$. Then $t^{\prime}$ is bounded by a constant, namely,

$$
\sqrt{\left(2 \mathbf{q}^{2} \exp [-\pi \alpha]+\operatorname{sexp}[-\pi \alpha / 2]\right)}
$$

while $z^{\prime}$ is always finite. If, on the other hand, $\beta<-2$ we see that $z^{\prime}<|\mathbf{q}| \exp [-\pi \alpha / 2]$ while $t^{\prime}$ is finite for finite $\lambda$.

\section{Non-space-like geodesics}

At this point we are in condition of writing a general form of the geodesics in the non-space-like case.

$$
\begin{gathered}
\frac{d t}{d \lambda}=\cosh w \quad \mathcal{F}(x, t) \mathcal{L}(x, t), \\
\frac{d x}{d \lambda}=\sinh w \quad \mathcal{F}(x, t) \mathcal{L}(x, t), \\
\mathcal{L}(x, t)=\frac{1}{|\mathbf{q}| \sqrt{2}}\left\{2 \mathbf{q}^{2}+2 \mathbf{p}^{2} \frac{[\sinh (\mu x / 2)]^{2(\beta+1)}[\cosh (\mu x / 2)]^{2(1-\beta)}}{[\sinh (\mu x)]^{2(\beta+2)}[\cosh (\mu t)]^{2(\beta+1)}}+\frac{\mathbf{s} e^{\alpha g d(\mu t)}}{[\cosh \mu t]^{\beta}[\cosh (\mu x / 2)]^{2 \beta}}\right\}^{1 / 2} .
\end{gathered}
$$

The evolution of $w$ in terms of the affine parameter can be obtained after some manipulations. Define the functions

$$
\begin{aligned}
& \Lambda_{1}(x, t)=e^{-2 \alpha g d(\mu t)}\left\{\mathbf{p}^{2} \frac{[\sinh (\mu x / 2)]^{2(1+\beta)}[\cosh (\mu x / 2)]^{2\left(1-\beta^{2}-\beta\right)}[\alpha+(\beta+2) \sinh \mu t]}{[\cosh \mu t]^{(2 \beta+5)}[\sinh \mu x]^{2(\beta+2)}},+\mathbf{q}^{2} \frac{\alpha-\beta \sinh \mu t}{[\cosh (\mu x / 2)]^{2 \beta^{2}}[\cosh \mu t]^{3}}\right\}, \\
& \Lambda_{2}(x, t)=e^{-2 \alpha g d(\mu t)}\left\{\frac{\mathbf{p}^{2}}{4} \frac{[\sinh (\mu x / 2)]^{2 \beta}[\beta-(\beta+2) \cosh \mu x]}{[\cosh (\mu x / 2)]^{2 \beta^{2}+2 \beta}[\sinh \mu x]^{2 \beta+3}[\cosh \mu t]^{2 \beta+4}}+\beta \mathbf{q}^{2} \frac{\sinh (\mu x / 2)}{[\cosh (\mu x / 2)]^{2 \beta^{2}+1}[\cosh \mu t]^{2}}\right\},
\end{aligned}
$$

then the equation for $w$ becomes

$$
\begin{aligned}
\frac{d w}{d \lambda}= & \cosh w \quad \mathcal{F}(x, t) \mathcal{L}(x, t) \\
& \times\left[-\frac{\mu}{2} \beta(\beta+1) \tanh \left(\frac{\mu x}{2}\right)-\frac{\mu \Lambda_{2}(x, t)}{[\mathcal{F}(x, t) \mathcal{L}(x, t)]}\right] \\
& +\sinh w \mathcal{F}(x, t) \mathcal{L}(x, t) \\
& \times\left[-\frac{\mu}{2} \frac{\alpha+(\beta+2) \sinh \mu t}{\cosh \mu t}+\frac{\mu \Lambda_{1}(x, t)}{[\mathcal{F}(x, t) \mathcal{L}(x, t)]}\right] .
\end{aligned}
$$

Equations (3.27) and (3.28) can be obtained, after some te- dious algebra, from Eqs. (3.1), (3.2) and (3.6), (3.7) following the same procedure outlined in the case of Eqs. (3.15), (3.16).

From these equations we see that the only possibly dangerous terms are the ones containing some hyperbolic sinus in the denominator. These terms could explode for $x \rightarrow 0$. We can see that this is not the case. Suppose we isolate the possible dangerous contribution by keeping only the leading terms in the limit $x \rightarrow 0$. We see that

$$
\lim _{x \rightarrow 0} \mathcal{F}(x, t) \mathcal{L}(x, t)=\mathcal{M}(x, t),
$$

where $\mathcal{M}(x, t)$ is simply given by Eq. (3.18). Recall now that by expressing $x^{\prime \prime}$ as $w^{\prime} t^{\prime}$ and $t^{\prime \prime}$ as $w^{\prime} t^{\prime}$, from Eqs. (3.5) and (3.1) we get an identity which allows to simplify, in the limit $x \rightarrow 0$, Eq. (3.28). The final result for Eq. (3.28) reproduces 
exactly Eqs. (3.17), (3.18). Therefore, the only possible singularities arising in the general case are the ones already appearing in the case of lightlike geodesics with $z^{\prime}=0$. We can repeat the same argument discussed in that context and conclude that the geodesics are complete also in this case, since the dangerous value (i.e., $x=0$ ) cannot be reached for finite value of $\lambda$. In different limits Eqs. (3.28) reproduce the various geodesics specifically studied in the previous subsections. We can say that the general case does not introduce unexpected singularities and we will finally conclude that the family of non-space-like geodesics is all complete. The derivatives of each coordinate are always finite for finite values of the affine parameter.

From the conclusions of the present section we see that the solution of the low-energy beta functions discussed in Ref. [13] and illustrated in Eq. (2.6) represent a class of singularity free backgrounds. In fact in Ref. [13] the regularity of the curvature invariants was proven by direct calculation of the scalar curvature and of the squares of all the other curvature invariants. In this paper we showed that all the non-space-like geodesics are complete. Given the (rather surprising) properties of these solutions of the effective action (2.1) we find useful to discuss in a more detailed fashion the interplay among our geometry and the singularity theorems.

\section{SINGULARITY THEOREMS AND DILATON-DRIVEN COSMOLOGIES}

From the results discussed in the previous section we can be confident that our space-time is geodesically complete and that, at the same time, the curvature invariants are everywhere defined in time and space without hitting any curvature singularity [13]. Thus we can conclude that our solutions of Eqs. (2.6) and (2.7) are singularity free.

Many questions can arise at this point. For example one could ask which hypotheses of the singularity theorems turn out to be violated, which are the asymptotics of our solutions and so on and so forth. Before addressing these questions we want to make a general statement. In the context of superstring inspired cosmological models it is quite important, from the physical point of view, to discuss the singularity properties of a model both in the string and in the Einstein frame. In the string frame the Planck length is not a constant and its time evolution is determined by the dilaton coupling. The true constant in the string frame is then the string length $\left(\lambda_{s}\right)$. Indeed in Ref. [13] it was shown that, for the class of models at hand, the regularity of the curvature invariants occurs both in the Einstein and in the string frame.

There are physical reasons to prefer the string to the Einstein frame. For example, the study of the motion of classical strings in cosmological backgrounds showed that the center of mass of the string follows geodesics with respect to the string frame $[25,26]$ but not with respect to the Einstein frame. Moreover, looking at the properties of the cosmological solutions of the low-energy beta functions it is well known that the superinflationary expansion appearing in the string frame description of the pre-big-bang scenario, turns into an accelerated contraction in the Einstein frame.

In this paper we discussed the completeness of the geo- desics in the string frame. It is not true, in general, that the geodesic completeness in the string frame implies the geodesics completeness in the Einstein frame. In our case using the fact that the dilaton coupling (appearing in the conformal factor) is completely regular and using also the fact that our metric is diagonal [27]the geodesic completeness can be shown to hold also in the Einstein frame and an explicit calculation (which we do not report) tells us that this is indeed the case.

In more general terms, the situation is not so obvious and the singularity properties of the solutions of the low-energy beta functions cannot be simply translated from the string to the Einstein frame. For example, it is almost intuitive that singular metrics in one frame can become regular in the other. Typically this can happen if the dilaton is evolving towards a phase of linear growth (in cosmic time). If this is the case, in spite of the regularity of the curvature invariants (say in the string frame), it is almost obvious that the singularity will reappear in the Einstein frame thanks to the conformal transformation involving the dilaton coupling. This is exactly what happens in most of the regular solutions of the low-energy beta functions [9] which are indeed only regular in the string frame. In the terminology of the present paper we call singularity free only those solutions which are regular and geodesically complete in the string frame and in all the other frames conformally related to it. We want to recall that in our examples the dilaton always evolves between two constant regimes, and, therefore, the regularity in one frame implies the regularity in the other.

Therefore, a well defined problem would be to precisely formulate the singularity theorems directly in the string frame. We will not address this quite important problem in general (even if it would be by itself of extreme interest). Following the approach adopted in the previous sections we will only be concerned with our solutions and we will postpone to future studies the considerations related to more general metrics (where, for instance, the dilaton is not going to constant value but either it oscillates or it increases linearly in cosmic time).

In this spirit a legitimate question to ask is, in our context if, given a non-space-like vector $u^{\mu}$, the condition

$$
u^{\mu} u^{\nu} R_{\mu \nu}>0
$$

is satisfied or not. In the context of the singularity theorems one often refers to the strong energy condition $\left(u^{\mu} u^{\nu} R_{\mu \nu}\right.$ $\geqslant 0$ for all causal vectors $u^{\mu}$ ) and to the null convergence condition $\left(v^{\mu} v^{\nu} R_{\mu \nu} \geqslant 0\right)$ for all null vectors $v^{\mu}$. We will try to avoid using the weak energy condition $\left(T_{\mu \nu} u^{\mu} u^{\nu} \geqslant 0\right.$ for all causal vectors $u^{\mu}$ ) and the dominant energy condition since they involve directly the energy-momentum tensor $T_{\mu \nu}$.

The singularity theorems usually invoke an appropriate boundary or initial condition which tries to express the fact that some finite portion of the space is trapped within itself and nothing can escape from it. Since historically the singularity theorems were firstly applied to the problem of the gravitational collapse [28], this condition is quite easy to understand physically. In a cosmological context this condi- 
tion might look at first sight more difficult to understand or be less motivated. Of course this is not the case, but, nonetheless the initial boundary condition is an absolute necessity for the proof of the singularity theorems in their strongest version (for a more detailed discussion of this point see the excellent review of Ref. [29]).

In more formal terms a trapped surface is a spacelike surface whose two (future directed) null fundamental forms have the same sign. If both traces are negative the surface is said to be future trapped, whereas, if they are both positive they are said to be past trapped.

In our solution something quite amusing happens. In fact it turns out that some of the models included in the class Eqs. (2.6) and (2.7) violate the condition given in Eq. (4.1). Some other models do not violate any causality or energy condition but do violate the initial boundary condition in the sense that closed surfaces are not necessarily trapped.

To show this consider the explicit calculation reported in Appendix B. We see that the condition (4.1) is not satisfied since the contraction of the Ricci tensor with two generic non-space-like vectors changes signs. However, if we choose $\beta=-4$ the condition (4.1) is satisfied. In fact for $\beta=-4$ the hyperbolic sinuses responsible for the sign flips in Eq. (4.1) disappear from the final expression. If $\beta=-4$ we can show that in spite of the fact that the condition of Eq. (4.1) is satisfied, the space-time does contain closed surfaces that are not trapped.

In the following we want to study the possible existence of closed surfaces which are not trapped. We will study this problem in the Einstein frame where the techniques of differential geometry can be safely employed [29]. In this frame our metric will be simply given by

$$
\begin{aligned}
G_{00} & =\mathcal{A}(x, t), \quad G_{x x}=-\mathcal{A}(x, t), \quad G_{y y} \\
& =-\mathcal{B}(x, t) \mathcal{C}(x, t), \quad G_{z z}=-\frac{\mathcal{B}(x, t)}{\mathcal{C}(x, t)},
\end{aligned}
$$

with

$$
\begin{aligned}
\mathcal{A}(x, t)= & {[\cosh \mu t]^{2+\beta}\left[\cosh \left(\frac{\mu x}{2}\right)\right]^{2 \beta(\beta+1)}, } \\
\mathcal{B}(x, t)= & \cosh \mu t \sinh \mu x, \\
\mathcal{C}(x, t)= & {[\cosh \mu t]^{1+\beta}\left[\sinh \left(\frac{\mu x}{2}\right)\right] } \\
& \times\left[\cosh \left(\frac{\mu x}{2}\right)\right]^{1+2 \beta} .
\end{aligned}
$$

The connection between the Einstein and the string frame is

$$
\Phi=\phi, \quad g_{\mu \nu}=e^{\Phi} G_{\mu \nu} .
$$

Given the metric (4.2), consider some closed twodimensional surface $S$ embedded in the our spece-time. In order to describe closed surfaces in the spce-time we have to specify the embedding equations, namely,

$$
x^{\mu}=\Lambda^{\mu}(u, v),
$$

where $u$ and $v$ are the intrinsic coordinates on $S$. For our calculation we will consider mostly two-dimensional spheres of constant radius $R$ which can be parametrized in terms of the embedding equations as

$$
\begin{aligned}
& t=\Lambda^{t}(u, v)=T \\
& x=\Lambda^{x}(u, v)=R \sin u \cos v, \\
& y=\Lambda^{y}(u, v)=R \sin u \sin v \\
& z=\Lambda^{z}(u, v)=R \cos u
\end{aligned}
$$

On the surface $S$ one can easily define the tangent vectors and the (two-dimensional) intrinsic metric

$$
\gamma_{a b}=\left.e_{a}^{\mu} e_{b}^{\nu} g_{\mu \nu}\right|_{S}, \quad e_{a}^{\mu}=\frac{\partial \Lambda^{\mu}}{\partial u^{a}}, \quad e_{b}^{\nu}=\frac{\partial \Lambda^{\mu}}{\partial u^{b}},
$$

where $a, b \cdots$ run over the intrinsic coordinates $u, v$, whereas $\mu, \nu \cdots=0,1,2,3$ run over the local coordinate chart $x^{\mu}=(t, x, y, z)$.

A nonzero one-form $\omega$ (defined on $S$ ) orthogonal to the tangent vectors (i.e., $\omega_{\mu} e_{u}^{\mu}=0$ and $\omega_{\mu} e_{v}^{\mu}=0$ ) is called normal one-form. In the case of the sphere (and more generally for a closed regular surface) there are indeed, at each point $(u, v)$ two normal one-forms, one pointing outside $S$ and the other pointing inside $S$. If it exists a normal one-form which is timelike both the two normal one-forms can be chosen to be null on the (spacelike) surface $S$. From the explicit form of the embedding equations (4.6) we can compute, in our metric, the two null normal one-forms $k^{ \pm}$orthogonal to the tangent vectors

$$
k_{\mu}^{ \pm} e_{a}^{\mu}=0, \quad k_{\mu}^{+} k^{+\mu}=0, \quad k_{\mu}^{-} k^{-\mu}=0, \quad k_{\mu}^{+} k^{-\mu}=1 .
$$

After some algebra we find that

$$
\begin{aligned}
& \sqrt{2} k^{+}=j(u, v, T)\{d t+p(u, v, T) \\
& \times[\sin u(\cos v d x+\sin v d y)+\cos u d z]\}\left.\right|_{S}, \\
& \sqrt{2} k^{-}=j(u, v, T)\{d t-[p(u, v, T) \sin u \\
& \times(\cos v d x+\sin v d y) \\
& +\cos u \quad q(u, v, T) d z]\}\left.\right|_{S},
\end{aligned}
$$

with

$$
\begin{aligned}
p(u, v, T)= & \left\{\mathcal{A}(u, v, T) \cos ^{2} u+\sin ^{2} u\right. \\
& \times\left[\mathcal{B}(u, v, T) \mathcal{C}(u, v, T) \cos ^{2} v\right. \\
& \left.\left.+\mathcal{A}(u, v, T) \sin ^{2} v\right]\right\}-1 / 2,
\end{aligned}
$$




$$
\begin{aligned}
q(u, v, T)= & 2\{\mathcal{A}(u, v, T)+\mathcal{B}(u, v, T) \mathcal{C}(u, v, T) \\
& +2 \mathcal{A}(u, v, T) \mathcal{C}(u, v, T)^{2} \\
& -[\mathcal{A}(u, v, T)+\mathcal{B}(u, v, T) \mathcal{C}(u, v, T) \\
& \left.-2 \mathcal{A}(u, v, T) \mathcal{C}^{2}(u, v, T)\right] \\
& \times \cos 2 u-2[\mathcal{A}(u, v, T) \\
& -\mathcal{B}(u, v, T) \mathcal{C}(u, v, T)] \\
& \left.\times \cos 2 v \sin ^{2} u\right\}^{-1 / 2}, \\
j(u, v, T)= & \sqrt{\mathcal{A}(u, v, T) \mathcal{B}(u, v, T) \mathcal{C}(u, v, T),}
\end{aligned}
$$

where $\mathcal{A}(u, v, T) \cdots$ stand for $\mathcal{A}[x(u, v), t(u, v)] \cdots$ as specified by the embedding equations (4.6). The notation $\left.\right|_{S}$ appearing in Eq. (4.9) [and in Eq. (4.7)] is meant to stress the fact that all the functions appearing in the definition of the two null normal one-forms have to be evaluated on the surface $S$. Needless to say that Eqs. (4.9), (4.10) satisfy all the requirements of Eq. (4.8) as it can be directly checked by repeated use of trigonometric identities.

For the two (future directed) normal one-form, the associated second fundamental forms can be defined as

$$
K_{a b}^{ \pm}=e_{b}^{\mu} e_{a}^{\nu} \nabla_{\nu} k_{\mu}^{ \pm},
$$

where $\nabla_{\nu}$ is the covariant derivative with respect to the metric $g_{\mu \nu}$. In order to show that there are closed surfaces which are not trapped we want to compute the two null (future directed) second fundamental forms $K_{a b}^{ \pm}$and their related traces

$$
K^{+}=\gamma^{a b} K_{a b}^{+}, \quad K^{-}=\gamma^{a b} K_{a b}^{-} .
$$

The spacelike surface $S$ is trapped if and only if the traces of the two null second fundamental forms have the same signs.

We want to show that in our case, namely in the case of the metric given by Eq. (4.2) regular closed surfaces are not trapped. What we will present is not a proof in the strict mathematical sense but it is more a plausibility argument. First of all we will take the two-spheres defined by the embedding equations (4.6) and we will compute the traces of the two second fundamental forms given in Eq. (4.12) and we will show that in some point of $S$ they do not have the same sign. We will then argue that one can expect a similar result for any closed surface defined through a regular embedding. Needless to stress that the second step of our plausibility argument needs to be more firmly justified with a formal mathematical proofs which we will not attempt in this paper. The spirit of our proof is, therefore, not general. The idea is to look for closed surfaces which are not trapped. We then take a two-sphere and we investigate if in any point of the surface the traces of the two second fundamental forms have the same sign. If this does not happen for any point, then we can conclude that there are closed surfaces which are not trapped simply because we found one of those surfaces with this property. Our proof is constructive in the sense that we show explicitly one closed surface which is not trapped.
Using Eqs. (4.5), (4.6) together with the general form of our metric we can compute easily the two-dimensional metric on the sphere: namely,

$$
\begin{aligned}
\gamma_{u u}= & -R^{2}\left[\cos ^{2} u \cos ^{2} v \mathcal{A}(u, v, T)\right. \\
& +\cos ^{2} u \sin ^{2} v \mathcal{B}(u, v, T) \mathcal{C}(u, v, T) \\
& \left.+\sin ^{2} u \frac{\mathcal{B}(u, v, T)}{\mathcal{C}(u, v, T)}\right], \\
\gamma_{v v}= & -R^{2} \sin ^{2} u\left[\sin ^{2} v \mathcal{A}(u, v, T)\right. \\
& \left.+\cos ^{2} v \mathcal{B}(u, v, T) \mathcal{C}(u, v, T)\right], \\
\gamma_{u v}= & \gamma_{v u}=\frac{R^{2}}{4} \sin 2 u \sin 2 v \\
& \times[\mathcal{A}(u, v, T)-\mathcal{B}(u, v, T) \mathcal{C}(u, v, T)] .
\end{aligned}
$$

Consider now the point $P$ where $|x|$ is maximal, namely the point $u=\pi / 2, v=0$ on the constant time hypersurface $t$ $=T$. Using Eq. (4.9) into Eq. (4.11) for our two-dimensional metric (4.13) defined on $S$ we find that the two traces of Eq. (4.12) at $P$ are, in the case $\beta=-4$

$$
\begin{aligned}
K_{P}^{ \pm}= & \frac{\mu}{2 \sqrt{2}}[\cosh (\mu T)]\left[\cosh \left(\frac{\mu R}{2}\right)\right]^{-11}\left[\sinh \left(\frac{\mu R}{2}\right)\right]^{-1} \\
& \times[\tanh \mu T \tanh \mu R \mp 1]
\end{aligned}
$$

We can clearly see that the sign of the product of the two traces is determined by

$$
K_{P}^{+} K_{P}^{-} \propto\left[\tanh ^{2} \mu T \tanh \mu R-1\right]<0,
$$

which is always negative. Then the two traces of the two null (forward directed) second fundamental forms have opposite sign in the point $P$ where $|x|$ is maximal. But a spacelike surface is trapped if and only if $K^{+} K^{-}$is positive everywhere on $S$. Since we found (at least) one point where this does not happen we conclude that in our spacetime there are no closed trapped spheres.

Let us consider now a generic closed (spacelike) surface with embedding equations (4.5) and suppose that it is trapped. Since the surface is compact it must also have a point where $|x|$ is maximal. We will denote, for simplicity, $\left|x_{\max }(u, v)\right|=R$ on a constant time hypersurface $t=T$. Therefore the normal at $P$ can be estimated (locally) as

$$
\begin{aligned}
K_{P}^{ \pm}= & g^{y y} \partial_{y} k_{y}(P)+g^{z z} \partial_{z} k_{z}(P)+\frac{\mu}{2 \sqrt{2}}[\cosh (\mu t)] \\
& \times\left[\cosh \left(\frac{\mu x}{2}\right)\right]^{-11}\left[\sinh \left(\frac{\mu x}{2}\right)\right]^{-1} \\
& \times[\tanh \mu t \tanh \mu x \mp 1],
\end{aligned}
$$

where $(x, t)$ are expressed in terms of the intrinsic coordinates $(v, u)$. An important property of a generic regular em- 
bedding is that the functions $\Phi^{\mu}(u, v)$ appearing in Eq. (4.5) should be continuous up to their third derivative, i.e., they should be $C^{3}$ in a more correct terminology (see Ref. [29] for a more precise definition). Now, since both $\partial_{y} k_{y}(P)$ and $\partial_{z} k_{z}(P)$ have (locally) opposite signs, respectively, for ingoing and outgoing normals, we have

$$
\begin{aligned}
K_{P}^{+} \leqslant & +\frac{\mu}{2 \sqrt{2}}[\cosh (\mu T)]\left[\cosh \left(\frac{\mu R}{2}\right)\right]^{-11}\left[\sinh \left(\frac{\mu R}{2}\right)\right]^{-1} \\
& \times[\tanh \mu T \tanh \mu R-1]<0, \\
K_{P}^{+} \geqslant & +\frac{\mu}{2 \sqrt{2}}[\cosh (\mu T)]\left[\cosh \left(\frac{\mu R}{2}\right)\right]^{-11}\left[\sinh \left(\frac{\mu R}{2}\right)\right]^{-1} \\
& \times[\tanh \mu T \tanh \mu R+1]>0 .
\end{aligned}
$$

Since the two traces have opposite signs our space-time admits closed surfaces which are not trapped. Thus the initial (boundary) condition of the singularity theorems is not satisfied and this is the reason why, also in the $\beta=-4$ case we are able to find nonsingular solutions.

We want to mention another interesting properties of our solutions. If we look at the curvature invariants we said that they are all bounded. Moreover a quite amusing fact holds. Namely, if we look at the ratio of the Weyl invariant compared to the Riemann invariant [13] we see that

$$
\lim _{x \rightarrow \infty, t \rightarrow \infty} \frac{C^{\mu \nu \alpha \beta} C_{\mu \nu \alpha \beta}}{R^{\mu \nu \alpha \beta} R_{\mu \nu \alpha \beta}}=\text { const. }
$$

This asymptotic behavior, together with the fact that $R_{\mu \nu} R^{\mu \nu}$ goes to zero (for $[x, t] \rightarrow \infty$ ) faster than $C^{\mu \nu \alpha \beta} C_{\mu \nu \alpha \beta}$ signals the fact that, in our context, the terms $\nabla_{\mu} \nabla_{\nu} \phi$ are asymptotically subleading if compared to the Weyl tensor (recall that, for our solutions, $R_{\mu \nu}=-\nabla_{\mu} \nabla_{\nu} \phi$ ).

\section{SPHERICALLY SYMMETRIC LITTLE BANGS?}

The solutions discussed in the previous sections have various symmetries: symmetries inherited from the lowenergy effective action (such as scale invariance and dilaton shift), accidental symmetries (such as parity) appearing in the solutions of the equations of motion and symmetries of the metric tensor. This last class of symmetries was imposed from the very beginning [13], by requiring that our geometry had two spacelike killing vector fields admitting an Abelian group $G_{2}$ on spacelike orbits $S_{2}$ [23]. A legitimate question to ask is what does it change when we assume from the very beginning a different symmetry of the metric. Recent studies [3] were dealing, for example, with spherically symmetric manifolds arguing that the singularity is a (rather generic) property of string cosmology. Moreover, those authors were more interested in the Einstein frame solutions that in the string frame ones (only for technical reasons). The question is then if it is possible to find regular and geodesically complete solutions of the low-energy beta functions in the Einstein frame with a spherically symmetric line element. This problem was recently discussed in the context of general relativity $[29,31]$. We present here a possible example in this direction.

We want to solve the low-energy beta functions with a spherically symmetric ansatz and we want to see if they admit regular solutions. Suppose that the metric is spherically symmetric and with line element

$$
d s^{2}=f(r, t)\left[d t^{2}-d r^{2}\right]-g(r, t)\left[d \theta^{2}+\sin ^{2} \theta d \varphi^{2}\right] .
$$

Assume then, following the techniques exploited of Ref. [13], that $f(r, t)$ and $g(r, t)$ can be factorized as the product of two regular functions only depending either on time or on space, namely,

$$
f(r, t)=e^{2 \mu r} \sigma(t), \quad g(r, t)=e^{2 \mu r} \rho(t) .
$$

Equations (2.2) and (2.3) can be easily written in the metric (5.1) with the ansatz (5.2). Since we want a completely homogeneous coupling we will set to zero the spatial derivatives of the dilaton:

$$
\begin{gathered}
2 \ddot{\phi}-\frac{\dot{\sigma}}{\sigma} \dot{\phi}+\left(\frac{\dot{\rho}}{\rho}\right)^{2}+\left(\frac{\dot{\rho}}{\rho}\right)\left(\frac{\dot{\sigma}}{\sigma}\right)+\left(\frac{\dot{\sigma}}{\sigma}\right)^{2} \\
-2 \frac{\ddot{\rho}}{\rho}-\frac{\ddot{\sigma}}{\sigma}+4 \mu^{2}=0
\end{gathered}
$$

$$
-\frac{\dot{\sigma}}{\sigma} \dot{\phi}-\frac{\dot{\rho}}{\rho} \frac{\dot{\sigma}}{\sigma}+\left(\frac{\dot{\sigma}}{\sigma}\right)^{2}-\frac{\ddot{\sigma}}{\sigma}=0 \quad(r r)
$$

$$
\frac{\ddot{\rho}}{\rho}+2 \frac{\sigma}{\rho}-\frac{\dot{\rho}}{\rho} \dot{\phi}-4 \mu^{2}=0 \quad(\theta \theta)
$$

$$
\begin{gathered}
\dot{\phi} \frac{\dot{\rho}}{\rho}-\frac{\ddot{\rho}}{\rho}-2 \frac{\sigma}{\rho}+4 \mu^{2}=0 \quad(\varphi \varphi), \\
2 \ddot{\phi}-\dot{\phi}^{2}+2 \dot{\phi} \frac{\dot{\rho}}{\rho}-2 \frac{\ddot{\rho}}{\rho}-\frac{\ddot{\sigma}}{\sigma}-2 \frac{\sigma}{\rho}+\frac{1}{2}\left(\frac{\dot{\rho}}{\rho}\right)^{2}+\left(\frac{\dot{\sigma}}{\sigma}\right)^{2} \\
+6 \mu^{2}=0 \quad(\phi) .
\end{gathered}
$$

From Eq. (5.4) we get, integrating once, that, $\sigma=\exp [\phi]$. The other equations give a consistent solution in the form

$$
\phi=2 \mu t-\log \left(1+2 e^{2 \mu t}\right)+\gamma, \quad \rho=\frac{e^{2 \mu t}}{2 \mu^{2}}, \quad \sigma=\frac{e^{2 \mu t}}{2 e^{2 \mu t}+1},
$$

notice that $\gamma$ is the integration constant associated with the evolution of the dilaton; the overdot denotes the derivation with respect to $t$. Since the action we are discussing is invariant under dilaton shift, we can require that the dilaton starts 
its evolution deeply in the perturbative regime where $\gamma$ will be very negative and $g(\phi) \ll 1$. The line element can then be written as

$$
d s^{2}=\frac{e^{2 \mu(r+t)}}{2 e^{2 \mu t}+1}\left[d t^{2}-d r^{2}\right]-\frac{e^{2 \mu(r+t)}}{2 \mu^{2}}\left[d \theta^{2}+\sin ^{2} \theta d \varphi^{2}\right] .
$$

We would be tempted to spot this as an example of a (spherically symmetric) little bang dynamics. This does not seem the case. In fact there are quite amusing features associated with this solution which makes it in a sense unphysical but also interesting, in another sense. The curvature invariants associated with this solution are all regular (see Appendix C for the results). In order to understand why this example is qualitatively different from the ones discussed in the previous sections we can look at the asymptotic behavior of the dilaton coupling:

$$
\lim _{t \rightarrow+\infty} \phi(t)=-2 \sqrt{2}, \quad \lim _{t \rightarrow-\infty} \phi(t)=2 \mu t .
$$

Thus $\dot{\phi}$ goes to zero for $t \rightarrow+\infty$ and it goes to a constant (i.e., $\dot{\phi} \sim 2 \mu$ ) for $t \rightarrow-\infty$. This behavior has to be contrasted with the one exhibited by the solutions reported in Sec. I where $\dot{\phi}$ was going to zero in both limits. From a physical point of view we would prefer a coupling evolving between two asymptotically constant regimes. However, one might speculate that a linearly evolving dilaton might be, after all, not so wrong especially in connection with the analysis of initial conditions in the pre-big-bang scenario $[3,8]$. The second nasty feature of the solution (see Appendix C for details) is that the curvature invariants are regular in space but they exponentially diverge in time (in the string frame) for $t \rightarrow$ $-\infty$. This is clearly a fatal feature for this solution. It is, however, interesting to notice that the curvature invariants are regular and well defined in the Einstein frame. The reason for this drastically different behavior in the two frames is the linear evolution of the dilaton (for $t \rightarrow-\infty$ ) which appears in the conformal factor defining the relation between the Einstein and the string frame. Unfortunately, looking at the Einstein frame picture, we cannot "see" the dynamics of a "little bang" since the curvature does not grow to a maximal value. On the other hand we see from the analytical form of the curvature invariants (Appendix C) that the curvature scalar decreases from a constant value.

From this example we draw two important conclusions. First of all, when dealing with an initial state with linear dilaton (as in Ref. [3]) regular solutions in the Einstein frame turn singular in the string frame. This does not exclude the interesting possibility that singular solutions (in the Einstein frame) can turn into spherically symmetric (regular) solutions in the string frame.

The second thing we learn from this example is that, apparently, the possibility of having a growing dilaton evolving from a constant value to another constant value (in such a way that the curvature invariants will be bounded and regular in both frames) seems to be associated to the cylindrical symmetry of the metric. We cannot say if this is a mere technical point (which helps in finding an analytical solution) or if it is somehow deeper than that. We hope to come back on this point in the near future.

We want finally to recall that the solution (5.9) is nothing but the string cosmological version of the one discussed in Refs. [29,31] (notice that in Ref. [31] other examples of homothetic cosmological space-times are given whereas in Ref. [29] the geodesics of this model are discussed).

\section{CONCLUSIONS AND SPECULATIONS}

In this paper we discussed the singularity properties of a class of solutions of the low-energy beta functions. The space-time we described turns out to be singularity free in the technical sense since it has the curvature invariants all bounded and since it is also geodesically complete. We discussed the peculiar features of our space-time in the light of the singularity theorems and we saw that at least one of their hypotheses was violated. In particular we found examples where the only condition to be violated is the existence of trapped (closed) surfaces. We also discussed the physical interpretation of this class of models. They describe, in a string cosmological context, the occurrence of an inhomogeneous phase with growing curvature and coupling. The dynamics of the model is driven by the kinetic energy of the dilaton coupling and the big-bang singularity is replaced by a maximal curvature phase which we named the little bang. The bang is associated with the maximal curvature which can be as large as the string curvature scale. The bang is small in the sense that the solutions never develop a singularity. Since the maximal curvature scale is always smaller than 1 (in string units) the tree level action is appropriate for the description of such a phase. These analytical examples show that it possible to regularize the singularities appearing in the low-energy beta functions with strong inhomogeneities. Our model describes, then, what we called inhomogeneous bangs. These examples also show that it is not a must to include corrections in the string tension expansion in order to have regular curvature invariants. We stress that, in our case, the geodesics are also complete and, therefore, no singularity appears in the geodesics for any finite value of the affine parameter. Since our solutions are defined from $t \rightarrow$ $-\infty$ to $t \rightarrow+\infty$ and since, for negative times, the dilaton coupling and the curvature are always increasing, it seems natural to find a contact between the models of inhomogeneous bang and the pre-big-bang models. Apparently the similarities are quite deep starting from the fact that the equations of motion of the fields in these two models are derived exactly from the same effective action.

Inhomogeneous pre-little-bang solutions can be also interesting in the context of the initial value problem in the prebig-bang scenario. One of the essential results of Ref. [3] was to connect the collapse of dilatonic waves (in the Einstein frame). The fact that the collapse was evolving towards a singularity has been regarded as a very good aspect since, it also meant a sufficiently long phase of Kasner regime and, ultimately, a quite long superinflationary phase (in the string frame). A natural question then arises: in which frame is the 
TABLE I. We report the Christoffel symbols computed in a geometry with two Abelian Killing vector fields which are hypersurface orthogonal and orthogonal to each other. The notations are the ones used in Sec. II.

$\Gamma_{x x}^{x}=\frac{1}{2}(\partial \log A / \partial x)$

$$
\Gamma_{y y}^{x}=-\frac{C B}{2 A}(\partial \log (B C) / \partial x)
$$

$\Gamma_{z z}{ }^{x}=-\frac{B}{2 A C}(\partial \log (B / C) / \partial x)$

$\Gamma_{x t}{ }^{x}=\frac{1}{2}(\partial \log A / \partial t)$

$\Gamma_{t t}^{x}=\frac{1}{2}(\partial \log A / \partial x)$

$\Gamma_{y t}{ }^{y}=\frac{1}{2}(\log (B C) / \partial t)$

$\Gamma_{z t}{ }^{z}=\frac{1}{2}(\partial \log (B / C) / \partial t)$

$\Gamma_{x y}{ }^{y}=\frac{1}{2}(\log (B C) / \partial x)$

$\Gamma_{x z}{ }^{z}=\frac{1}{2}(\partial \log (B / C) / \partial x)$

$\Gamma_{x x}{ }^{t}=\frac{1}{2}(\partial \log A / \partial t)$

$\Gamma_{y y}{ }^{t}=\frac{C B}{2 A}(\partial \log (C B) / \partial t)$

$\Gamma_{z z}{ }^{t}=\frac{B}{2 A C}(\partial \log (B / C) / \partial t)$

$\Gamma_{x t}{ }^{t}=\frac{1}{2}(\partial \log A / \partial x)$

$\Gamma_{t t}{ }^{t}=\frac{1}{2}(\partial \log A / \partial t)$

singularity "real"? Is it the Einstein frame (as argued in Ref. [3]) or is it the string frame? In other words where the singularity needs to be regularized. Our answer is, in both frames. The spherically symmetric examples reported in the present section are quite interesting in this respect. Our conclusion, moreover, is that the Hawking-Penrose theorems should be directly formulated in the string frame. This could be a great help also for the analysis of the intial condition problem.

We stress that our solutions are always inhomogeneous. The physical picture we have in mind is therefore the following. We would like to find a model where a phase of (homogeneous) growing dilaton coupling is replaced around $[t, x]$ $=0$ by an inhomogeneous phase. No explicit models of this kind have been constructed, up to now. At the same time recent investigations [30] showed that the occurrence of these types of models is not excluded (in a general relativity context) since explicit general families of solutions were constructed where the ordinary Friedmann-RobertsonWalker models appear together with other singularity-free (inhomogeneous) perfect fluid models. Our context is certainly different, but, nonetheless, we think that it might be useful to investigate the possible occurrence of such a scenario in a superstring cosmological framework.

\section{ACKNOWLEDGMENTS}

I would like to thank J. M. M. Senovilla for very useful communications and G. Veneziano for kind interest in this investigation. I would also like to thank the Theory Division of CERN (where part of this work was completed) for financial support.

\section{APPENDIX A: CHRISTOFFEL SYMBOLS}

We report, in this appendix the Christoffel symbols computed in the case of the metric given in Eq. (2.6). In Table I we report the general expression of the Christoffel connections in the case of a metric with two Abelian Killing vectors which are hypersurface orthogonal and orthogonal to each others. From the expressions given in Table I, using the ex- plicit form of our solutions given in Eq. (2.6) we get, after some simple algebra,

$$
\begin{aligned}
& \Gamma_{t t}^{t}=\frac{\mu}{2} \frac{\alpha+(\beta+2) \sinh \mu t}{\cosh \mu t}, \quad \Gamma_{x x}{ }^{t}=\Gamma_{t t}{ }^{t}, \\
& \Gamma_{x t}{ }^{t}=\frac{\mu}{2} \beta(\beta+1) \tanh \left(\frac{\mu x}{2}\right), \quad \Gamma_{t x}{ }^{t}=\Gamma_{x t}{ }^{t}, \\
& \Gamma_{y y}{ }^{t}=\mu \frac{[\alpha+(\beta+2) \sinh \mu t]}{[\sinh (\mu x / 2)]^{-2}[\cosh (\mu x / 2)]^{2 \beta^{2}-2} \cosh \mu t}, \\
& \Gamma_{z z}{ }^{t}=\mu \frac{\alpha-\beta \sinh \mu t}{[\cosh (\mu x / 2)]^{4 \beta+2 \beta^{2}}[\cosh \mu t]^{3+2 \beta}}, \\
& \Gamma_{x x}^{x}=\frac{\mu}{2} \beta(\beta+1) \tanh \left(\frac{\mu x}{2}\right), \quad \Gamma_{t t}^{x}=\Gamma_{x x}^{x} \\
& \Gamma_{x t}{ }^{x}=\frac{\mu}{2} \frac{\alpha+(\beta+2) \sinh \mu t}{\cosh \mu t}, \quad \Gamma_{t x}{ }^{x}=\Gamma_{x t}{ }^{x}, \\
& \Gamma_{y y}{ }^{x}=\frac{\mu}{2}\left[\sinh \left(\frac{\mu x}{2}\right)\right] \frac{[\beta-(\beta+2) \cosh \mu x]}{[\cosh (\mu x / 2)]^{2 \beta^{2}-1}} \\
& \Gamma_{z z} x=\mu \beta \frac{\tanh (\mu x / 2)}{[\cosh (\mu x / 2)]^{4 \beta+2 \beta^{2}}[\cosh \mu t]^{2(\beta+1)}}, \\
& \Gamma_{y x}{ }^{y}=\frac{\mu}{2} \frac{(\beta+2) \cosh \mu x-\beta}{\sinh \mu x}, \quad \Gamma_{x y}{ }^{y}=\Gamma_{y x}{ }^{y}, \\
& \Gamma_{y t}{ }^{y}=\frac{\mu}{2} \frac{\alpha+(\beta+2) \sinh \mu t}{\cosh \mu t}, \quad \Gamma_{t y}{ }^{y}=\Gamma_{y t}{ }^{y}, \\
& \Gamma_{x z}^{z}=-\frac{\mu}{2} \beta \tanh \left(\frac{\mu x}{2}\right), \quad \Gamma_{z x}^{z}=\Gamma_{x z}^{z}, \\
& \Gamma_{z t}^{z}=\frac{\mu}{2}\left[\frac{\alpha-\beta \sinh \mu t}{\cosh \mu t}\right], \quad \Gamma_{t z}^{z}=\Gamma_{z t}^{z}
\end{aligned}
$$

From Eq. (2.6) the general form of the geodesics can be derived:

$$
\begin{aligned}
\frac{d^{2} t}{d \lambda^{2}}+ & \frac{1}{2} \frac{\dot{A}}{A}\left[\left(\frac{d t}{d \lambda}\right)^{2}+\left(\frac{d x}{d \lambda}\right)^{2}\right] \\
+ & \frac{A^{\prime}}{A} \frac{d t}{d \lambda} \frac{d x}{d \lambda}+\frac{\dot{B} C-\dot{C} B}{2 A C^{2}}\left(\frac{d z}{d \lambda}\right)^{2} \\
+ & \frac{\dot{B} C+\dot{C} B}{2 A}\left(\frac{d y}{d \lambda}\right)^{2}=0,
\end{aligned}
$$




$$
\begin{aligned}
\frac{d^{2} x}{d \lambda^{2}}+ & \frac{1}{2} \frac{A^{\prime}}{A}\left[\left(\frac{d t}{d \lambda}\right)^{2}+\left(\frac{d x}{d \lambda}\right)^{2}\right] \\
& +\frac{\dot{A}}{A} \frac{d t}{d \lambda} \frac{d x}{d \lambda}-\frac{B^{\prime} C-C^{\prime} B\left(\frac{d z}{2 A C^{2}}\right)^{2}}{d \lambda} \\
& -\frac{B^{\prime} C+C^{\prime} B}{2 A}\left(\frac{d y}{d \lambda}\right)^{2}=0 \\
\frac{d^{2} y}{d \lambda^{2}}+\left(\frac{\dot{B}}{B}+\frac{\dot{C}}{C}\right) \frac{d t}{d \lambda} \frac{d x}{d \lambda} & +\left(\frac{B^{\prime}}{B}+\frac{C^{\prime}}{C}\right) \frac{d x}{d \lambda} \frac{d y}{d \lambda}=0 \\
& +\left(\frac{B^{\prime}}{B}-\frac{C^{\prime}}{C}\right) \frac{d z}{d \lambda} \frac{d x}{d \lambda}=0 \\
\frac{d^{2} z}{d \lambda}+\left(\frac{\dot{B}}{B}-\frac{\dot{C}}{C}\right) \frac{d z}{d \lambda} \frac{d t}{d \lambda} & -\left(\frac{d x}{d \lambda}\right)^{2}=\frac{B C}{A}\left(\frac{d y}{d \lambda}\right)^{2}+\frac{B}{C A}\left(\frac{d z}{d \lambda}\right)^{2}+\frac{\mathbf{s}}{A}
\end{aligned}
$$

Notice that (only in this formula) we denoted with an overdot the derivation with respect to $t$ and with a prime the derivation with respect to $x$. In the rest of the paper the prime denotes simply the derivation with respect to the affine parameter $\lambda$. We apologize to the reader for the possible confusion.

\section{APPENDIX B: GEODESIC CONVERGENCE}

The geodesic convergence condition tells us that given any non-space-like vector $u$ we have that

$$
u^{\mu} u^{\nu} R_{\mu \nu}>0
$$

We will show that this condition is very important and interesting for the class of solutions we are discussing. In fact we can show that not for all choices of the parameter $\beta$ this condition is satisfied. In order to show this let us report the components of the Ricci tensor

$$
\begin{aligned}
R_{x}^{x}= & -\frac{\alpha \mu^{2}}{2} \frac{[\alpha+2 \sinh \mu t+\beta \sinh \mu t]}{[\cosh (\mu x / 2)]^{2 \beta(\beta+1)}[\cosh \mu t]^{2+\beta}} e^{-\alpha g d(\mu t),} \\
R_{y}^{y}= & -\frac{\alpha \mu^{2}}{2} \frac{[\alpha+\beta \sinh \mu t]}{[\cosh (\mu x / 2)]^{2 \beta(\beta+1)}[\cosh \mu t]^{4+\beta}} e^{-\alpha g d(\mu t)}, \\
R_{z}^{z}= & -\frac{\alpha \mu^{2}}{2} \frac{[\alpha-\beta \sinh \mu t]}{[\cosh (\mu x / 2)]^{2 \beta(\beta+1)}[\cosh \mu t]^{4+\beta}} e^{-\alpha g d(\mu t)} \\
R_{x}^{0}= & \frac{\alpha \beta(1+\beta) \mu^{2}}{2}\left[\sinh \left(\frac{\mu x}{2}\right)\right]\left[\cosh \left(\frac{\mu x}{2}\right)\right]^{-1-2 \beta(1+\beta)} \\
& \times[\cosh \mu t]^{-3-\beta} e^{-\alpha g d(\mu t)},
\end{aligned}
$$

$$
R_{0}^{0}=\frac{\mu^{2}}{2} \frac{\left[\beta^{2}-4+\alpha(4+\beta) \sinh \mu t\right]}{[\cosh \mu t]^{4+\beta}[\cosh (\mu x / 2)]^{2 \beta(\beta+1)}} e^{-\alpha g d(\mu t)} .
$$

Notice that something very amusing happens from this formulas. We point out that our class of exact solutions contains particular solutions where the geodesic convergence condition is clearly satisfied. Take, for example, $\alpha=2 \sqrt{3}$, then, since $\beta= \pm \sqrt{\alpha^{2}+4}$ we have that, for the value $\beta$ $=-\sqrt{\alpha^{2}+4} u^{\mu} u^{\nu} R_{\mu \nu}>0$. Of course in the opposite case (i.e., $\beta=4$ ) there is no definite sign for $u^{\mu} u^{\nu} R_{\mu \nu}$. As a consequence, since the geodesic convergence condition changes sign as an hyperbolic cosine the geodesic convergence will not occur.

It is interesting to notice that the geodesic convergence condition is always completely satisfied when translated to the Einstein frame where the low-energy beta functions can also be written and solved [13].

The Einstein frame is defined by the conformal transformation which diagonalizes the gravitational action by decoupling the dilaton from the Einstein-Hilbert Lagrangian. In the Einstein frame the Planck scale $\left[\lambda_{P} \sim 10^{-33} \mathrm{~cm}\right.$ is constant whereas the string scale evolves according to $\lambda_{s}(t)$ $=e^{-\phi / 2} \lambda_{P}$.] Since (in four dimensions) the Einstein and string frame dilatons are exactly equal, the string $\left(g_{\mu \nu}\right)$ and Einstein $\left(G_{\mu \nu}\right)$ metrics are connected by the obvious conformal rescaling $g_{\mu \nu}=e^{\phi} G_{\mu \nu}$. Notice that this conformal rescaling leads to changes in the geodesic convergence condition given in Eq. (B1). In fact in the Einstein frame we obtain that

$$
\mathcal{R}_{0}^{0}=\frac{\beta^{2}-4}{2} \mu^{2}[\cosh \mu t]^{-4-\beta}\left[\cosh \left(\frac{\mu x}{2}\right)\right]^{-2 \beta(\beta+1)}
$$

( $\mathcal{R}_{\mu}^{\nu}$ is the Ricci tensor in the Einstein frame). From this last equation we conclude that the geodesic convergence condition is satisfied without restrictions (in the Einstein frame) since the physical range of $\beta$ is $|\beta|>2$. This simple exercise can also show that the formulation of the singularity theorems in the string and Einstein frames can be different.

\section{APPENDIX C: A SPHERICALLY SYMMETRIC EXAMPLE}

In this section of the Appendix we report the curvature invariants associated with the spherically symmetric solution of the low-energy beta functions discussed in Sec. V. In the string frame the curvature invariants, computed from Eq. (5.10) are

$$
\begin{gathered}
R^{\mu \nu} R_{\mu \nu}=8 \mu^{4} \frac{1+4 e^{2 \mu t}+8 e^{4 \mu t}}{\left[1+2 e^{2 \mu t}\right]^{2}} e^{-4 \mu(r+t),} \\
R^{\mu \nu \alpha \beta} R_{\mu \nu \alpha \beta}=16 \mu^{4} \frac{1+4 e^{2 \mu t}+8 e^{4 \mu t}}{\left[1+2 e^{2 \mu t}\right]^{2}} e^{-4 \mu(r+t),}
\end{gathered}
$$




$$
\begin{aligned}
C^{\mu \nu \alpha \beta} C_{\mu \nu \alpha \beta} & =\frac{16 \mu^{4}}{3\left[2 e^{2 \mu t}+1\right]^{2}} e^{-4 \mu(r+t),} \\
R^{2} & =\frac{16 \mu^{4}}{\left[2 e^{2 \mu t}+1\right]^{2}} e^{-4 \mu(r+t)} .
\end{aligned}
$$

As we can see the curvature invariants explode exponentially for $t \rightarrow-\infty$. It is instructive to compute the curvature invariants also in the Einstein frame metric. They are

$$
\begin{gathered}
\mathcal{R}^{2}=\frac{4 \mu^{4}}{e^{4 \mu r}\left[1+2 e^{2 \mu t}\right]^{4}}, \\
\mathcal{R}_{\mu \nu} \quad \mathcal{R}^{\mu \nu}=\frac{4 \mu^{4}}{e^{4 \mu r}\left[1+2 e^{2 \mu t}\right]^{4}},
\end{gathered}
$$

$$
\begin{aligned}
\mathcal{R}_{\mu \nu \alpha \beta} \mathcal{R}^{\mu \nu \alpha \beta} & =\frac{12 \mu^{4}}{e^{4 \mu r}\left[1+2 e^{2 \mu t}\right]^{4}}, \\
\mathcal{C}_{\mu \nu \alpha \beta} \mathcal{C}^{\mu \nu \alpha \beta} & =\frac{16 \mu^{4}}{3 e^{4 \mu r}\left[1+2 e^{2 \mu t}\right]^{4}} .
\end{aligned}
$$

We clearly see from these equations that the curvature invariants are regular. However, something unpleasant happens: for $t \rightarrow-\infty$ the invariants do not tend to zero but to a constant. Notice that one of the main point of the examples discussed in Ref. [13] was that both in the Einstein and in the string frame the curvature invariants were going to zero for $t \rightarrow \pm \infty$. The peculiar behavior of our spherically symmetric example seems not completely physical and impossible to associate with a little ban in the sense discussed in this paper.
[1] A. Guth, Phys. Rev. D 23, 347 (1981); A. Linde, Phys. Lett. 108B, 389 (1982); A. Albrecht and P.J. Steinhardt, Phys. Rev. Lett. 48, 1220 (1982); A. Linde, Phys. Lett. 129B, 177 (1983); A. Linde, Phys. Rev. D 49, 748 (1994).

[2] S.W. Hawking and R. Penrose, Proc. R. Soc. London A314, 529 (1970); S. W. Hawking and G. F. R. Ellis, The Large Scale Structure of the Space-time (Cambridge University Press, Cambridge, England, 1973).

[3] G. Veneziano, Phys. Lett. B 265, 287 (1991); 406, 297 (1997); J. Maharana, E. Onofri, and G. Veneziano, J. High Energy Phys. 4, 4 (1998); A. Buonanno, T. Damour, and G. Veneziano, hep-th/9806230.

[4] C. Lovelace, Phys. Lett. 135B, 75 (1984); E.S. Fradkin and A.A. Tseytlin, Nucl. Phys. B261, 1 (1985); C.G. Callan et al., ibid. B261, 593 (1985); A. Sen, Phys. Rev. Lett. 55, 1846 (1985).

[5] K.A. Meissner and G. Veneziano, Phys. Lett. B 267, 33 (1997); Mod. Phys. Lett. A 6, 3397 (1991); M. Gasperini, J. Maharana, and G. Veneziano, Phys. Lett. B 272, 277 (1991); M. Gasperini and G. Veneziano, ibid. 277, 256 (1992); K. A. Meissner, ibid. 392, 298 (1997); A. Sen, ibid. 271, 295 (1991); S. Hassan and A. Sen, Nucl. Phys. B375, 103 (1992).

[6] M. Gasperini and M. Giovannini, Phys. Rev. D 47, 1519 (1993); M. Gasperini, M. Giovannini and G. Veneziano, Phys. Rev. Lett. 75, 3796 (1995); R. Brustein, M. Gasperini, M. Giovannini and G. Veneziano, Phys. Lett. B 361, 45 (1995); M. Giovannini, Phys. Rev. D 56, 3198 (1997); 56, 631 (1997); 55, 595 (1997).

[7] E. Kiritsis and K. Kounnas, Nucl. Phys. B (Proc. Suppl.) 41, 311 (1997).

[8] M. Turner and E. Weinberg, Phys. Rev. D 56, 4604 (1997); A. Linde, talk delivered at the Workshop on Cosmology and Particle Physics (CAPP 98), Geneva, Switzerland, 1998 (unpublished).

[9] M. Gasperini and M. Giovannini, Phys. Lett. B 287, 56 (1992); R. Brustein and G. Veneziano, ibid. 329, 429 (1994); I. Antoniadis, J. Rizos, and K. Tamwakis, Nucl. Phys. B415, 497
(1993); R. Easther, K. Maeda and D. Wands, Phys. Rev. D 53, 4247 (1996); S.J. Rey, Phys. Rev. Lett. 77, 1929 (1996); M. Gasperini and G. Veneziano, Phys. Lett. B 387, 715 (1996); M. Gasperini, M. Maggiore, and G. Veneziano, Nucl. Phys. B494, 315 (1997).

[10] M. Gasperini and G. Veneziano, Astropart. Phys. 1, 317 (1993).

[11] M. Gasperini, J. Maharana and G. Veneziano, Phys. Lett. B 296, 51 (1997).

[12] J. Barrow and K. Kunze, Phys. Rev. D 55, 623 (1997); 56, 741 (1997).

[13] M. Giovannini, Phys. Rev. D 57, 7223 (1998).

[14] A. Vilenkin, Phys. Rev. D 46, 2355 (1992).

[15] A. Borde and A. Vilenkin, Int. J. Mod. Phys. D 5, 813 (1996); Phys. Rev. D 56, 717 (1997).

[16] J.M.M. Senovilla, Phys. Rev. Lett. 64, 2219 (1990).

[17] F.J. Chinea, L. Fernández-Jambrina, and J.M.M. Senovilla, Phys. Rev. D 45, 481 (1992).

[18] C.G. Hewitt, J. Wainwright and M. Glaum, Class. Quantum Grav. 8, 1505 (1991); L.K. Patel and X. Dadhich, ibid. 10, L85 (1993); E. Ruiz and J.M.M. Senovilla, Phys. Rev. D 45, 1995 (1992); M. Mars, ibid. 51, R3989 (1995); L. FernaándezJambrina, Class. Quantum Grav. 14, 3407 (1997).

[19] M. Carmeli, Ch. Charach, and S. Malin, Phys. Rep. 76, 80 (1981); J. Wainwright, W.C. Ince, and B.J. Marshman, Gen. Relativ. Gravit. 10, 259 (1979); J. Wainwright, J. Phys. A 14, 1131 (1981); C.G. Hewitt and J. Wainwright, Class. Quantum Grav. 7, 2295 (1990); E.P. Liang, Astrophys. J. 204, 235 (1976); J. Wainwright and S.W. Goode, Phys. Rev. D 22, 1906 (1980); E. Verdaguer, Phys. Rep. 229, 1 (1993).

[20] I. S. Gradshteyn and I. M. Ryzhik, Tables of Integrals, Series and Products, 5th ed. (Academic, New York, 1994), p. 51.

[21] A. Krasinski, Inhomogeneous Cosmological Models (Cambridge University Press, Cambridge, England, 1997).

[22] E.J. Copeland, A. Lahiri, and D. Wands, Phys. Rev. D 50, 4868 (1994); K. Behrndt and S. Forste, Nucl. Phys. B430, 441 (1994); J. Levin, Phys. Rev. D 51, 462 (1995); 51, 1536 
(1995); E.J. Copeland, A. Lahiri, and D. Wands, ibid. 51, 1569 (1995); J. Levin, Phys. Lett. B 343, 69 (1995); J.E. Lidsey, Phys. Rev. D 55, 3303 (1997); A. Lukas, B.A. Ovrut, and D. Waldram, Nucl. Phys. B495, 365 (1997); M. Gasperini, M. Giovannini, K. Meissner, and G. Veneziano, in String Theory in Curved Space Times, edited by N. Sanchez (World Scientific, Singapore, 1998), p. 49.

[23] D. Kramer, H. Stephani and E. Herlt, Exact Solutions of Einstein's Field Equations (Cambridge University Press, Cambridge, England, 1980).
[24] F. Brauer and J. A. Nohel, The Qualitative Theory of Ordinary Differential Equations (Benjamin, New York, 1969).

[25] H.J. de Vega and N. Sanchez, Phys. Lett. B 197, 320 (1987).

[26] N. Sanchez and G. Veneziano, Nucl. Phys. B333, 253 (1990).

[27] H.S. Zia, Gen. Relativ. Gravit. 19, 465 (1987).

[28] R. Penrose, Phys. Rev. Lett. 14, 57 (1965).

[29] J.M.M. Senovilla, Gen. Relativ. Gravit. 30, 701 (1998).

[30] J.M.M. Senovilla, Phys. Rev. D 53, 1799 (1996).

[31] J. Carot and A.M. Sintes, Class. Quantum Grav. 14, 1183 (1997). 